\title{
Higher order spectra from an initially anisotropic universe
}

\author{
Masato Minamitsuji \\ Yukawa Institute for Theoretical Physics, Kyoto University, Kyoto, 606-8502, Japan
}

(Dated: June 15, 2018)

\begin{abstract}
In this paper, we present the higher order spectra of a scalar field produced through the higher derivative interactions in the initially anisotropic universe. Although we ignore the backreaction of the scalar field on the geometry, our analysis should have much overlap with the quantum fluctuations of the inflaton field in the anisotropic universe. We also include the planar modes whose momenta are along the plane which is perpendicular to the primordial preferred direction, for which effects of the initial anisotropy are not suppressed. The presence of a negative frequency mode produces features distinguishable from the case of the de Sitter inflation. We also show that richer features appear in the trispectra due to the primordial anisotropy.
\end{abstract}

PACS numbers: $98.80 . \mathrm{Cq}$

\section{INTRODUCTION}

Recent measurements by the WMAP satellite [1, 2] have suggested that the observed map of CMB anisotropy is almost consistent with the Gaussian and statistically isotropic primordial fluctuations from inflation. However, the issues on several anomalies in the recent data of the large-angle CMB temperature map have been controversial (see, e.g., [3-6]). These observational facts have motivated us to investigate the possibilities of the preinflationary anisotropy [7-12]. The cosmic no-hair theorem ensures that, in the presence of a positive cosmological constant, an initially anisotropic universe exponentially approaches the de Sitter spacetime under the strong or dominant energy condition [13]. Therefore, the initial universe may be highly anisotropic, and its consequences may be inherited in the cosmic observables today, such as CMB anisotropy, if the number of the e-foldings for inflation takes a minimal value 7 [10. In an expanding Kasner universe, one of two polarizations is coupled with the scalar mode, while the other is decoupled, leading to an asymmetry between them. There could be a difference between the amplitudes of gravitational waves due to the primordial anisotropy [11].

In this paper, first, we will investigate the bispectra of a scalar field in the initially anisotropic universe approaching the de Sitter spacetime (see, e.g., [14, 15] for reviews in the case of the standard inflation). Amoung two planar branches of the expanding Kasner spacetime, the adiabatic vacuum can be well defined in the branch where the initial spacetime structure is the product of the two-dimensional Milne universe and two-dimensional Euclidean space. Thus, the inflaton fluctuations are quantized in an adiabatic vacuum state different from the Bunch-Davies vacuum [16], which could give a signature distinguishable from the primordial anisotropy. Another important effect from the primordial anisotropy is the mixing of the scalar and tensor metric perturbations in terms of the three-dimensional symmetry 7 -10]. In particular, in the nonlinear perturbations such effects may be more significant. The purpose of this paper is to clarify the effects of the change of the initial vacuum state onto the higher order spectra in a single scalar field theory [10], and hence we will not discuss the coupling effects in the metric perturbations. Although we ignore the backreaction onto the geometry, our analysis should have much overlap with the cosmological perturbations. We can expect that some features appear in the limit $k_{1}+k_{2} \sim k_{3}$, which is expected to be sensitive to the mixture of a negative frequency mode [17]. The recent work Ref. [12] has confirmed part of the above expectations. The work focused on the nonplanar high-momentum modes whose momentum vectors are not along the plane where the effects of the negative frequency mode are suppressed due to the adiabaticity parameter [10, 11]. We expect that the nonnegligible contributions of the primordial anisotropy to the bispectrum would appear more significantly in the planar modes whose momenta are along the plane. Thus, our analysis includes them. We will also investigate the trispectra in which the contributions from the primordial anisotropy may become important even for the nonplanar modes.

The construction of this paper is as follows: In Sec. 2, we review the framework to analyze the higher order spectra in the anisotropic universe. In Sec. 3, we introduce the Kasner-de Sitter spacetime which exhibits an isotropization due to the presence of a positive cosmological constant. We also review the quantization of a free scalar field in this spacetime. In Sec. 4, we compute the bispectra in the presence of the higher order time-derivative interactions. Similarly, we investigate the trispectra in Sec. 5. In Sec. 6, we shall close this paper after giving a brief summary. 


\section{SCALAR FIELD INTERACTIONS IN THE ANISOTROPIC UNIVERSE}

\section{A. Canonical formulation}

In this section, we present the framework to analyze the higher order spectra of a scalar field in the anisotropic universe, based on the so-called in-in formalism [18].

We consider a scalar field in a curved spacetime background

$$
S_{\phi}=\int d^{4} x \sqrt{-g} \mathcal{L}=-\int d^{4} x \sqrt{-g}\left[\frac{1}{2}(\partial \phi)^{2}+V\right],
$$

where $V$ represents the interaction terms which are of higher order in terms of the scalar field and its derivatives. The form of $V$ will be specified in Sec. III. C. As the background spacetime, we consider an anisotropic universe with the planar symmetry

$$
d s^{2}=-d \tau^{2}+a(\tau)^{2} d x^{2}+b(\tau)^{2}\left(d y^{2}+d z^{2}\right)=-e^{6 \alpha(t)} d t^{2}+a(t)^{2} d x^{2}+b(t)^{2}\left(d y^{2}+d z^{2}\right),
$$

where $e^{\alpha}=\left(a b^{2}\right)^{\frac{1}{3}}$ is the avegaged scale factor and $d t=d \tau / e^{3 \alpha}$. We call the $(y, z)$-plane the planar direction, and simply denote the components of any vector in this direction by the symbol " $\perp$." We assume the universe approaches an isotropic one, $a \rightarrow b$, in the late time limit. Then the action of the scalar field Eq. (1) reduces to

$$
S_{\phi}=-\frac{1}{2} \int d t \int d^{3} x e^{6 \alpha}\left[-e^{-6 \alpha} \dot{\phi}^{2}+\frac{1}{a^{2}} \phi_{, x}^{2}+\frac{1}{b^{2}} \phi_{, \perp}^{2}+2 V\right],
$$

where we have defined the short-hand notation as $\dot{\phi}:=\phi_{, t}$ and $\phi_{, \perp}^{2}:=\phi_{, y}^{2}+\phi_{, z}^{2}$. We use the coordinate $t$ to label time rather than the physical time $\tau$. The conjugate momentum to the scalar field is given by $\pi_{\phi}=\frac{\delta S_{\phi}}{\delta \dot{\phi}}=\dot{\phi}$. The scalar field and conjugate momentum satisfy the equal time commutation relations $\left[\phi\left(t, x^{i}\right), \pi_{\phi}\left(t, y^{i}\right)\right]=i \delta^{(3)}\left(x^{i}-y^{i}\right)$ and $\left[\phi\left(t, x^{i}\right), \phi\left(t, y^{i}\right)\right]=\left[\pi_{\phi}\left(t, x^{i}\right), \pi_{\phi}\left(t, y^{i}\right)\right]=0$. The Hamiltonian density is defined by

$$
\mathcal{H}=\dot{\phi} \pi_{\phi}-\mathcal{L}=\frac{e^{6 \alpha}}{2}\left(e^{-6 \alpha} \pi_{\phi}^{2}+\frac{1}{a^{2}} \phi_{, x}^{2}+\frac{1}{b^{2}} \phi_{, \perp}^{2}+2 V\right) .
$$

The Hamiltonian is given by $H\left[\phi(t), \pi_{\phi}(t) ; t\right]:=\int d^{3} x \mathcal{H}$. The equation of motion for the scalar field is given by

$$
\dot{\phi}=i\left[H\left[\phi(t), \pi_{\phi}(t) ; t\right], \phi\right], \quad \dot{\pi}_{\phi}=i\left[H\left[\phi(t), \pi_{\phi}(t) ; t\right], \pi_{\phi}\right] .
$$

The solutions at $t$ can be expressed in terms of a similar operator at a very early time $t_{0}$ through the unitality transformation

$$
\phi(t)=U^{-1}\left(t, t_{0}\right) \phi\left(t_{0}\right) U\left(t, t_{0}\right), \quad \pi_{\phi}(t)=U^{-1}\left(t, t_{0}\right) \pi_{\phi}\left(t_{0}\right) U\left(t, t_{0}\right),
$$

where $U\left(t, t_{0}\right)$ obeys the differential equation

$$
\frac{d}{d t} U\left(t, t_{0}\right)=-i H\left[\phi(t), \pi_{\phi}(t) ; t\right] U\left(t, t_{0}\right) .
$$

The initial condition is given by $U\left(t_{0}, t_{0}\right)=1$. Let us briefly discuss the choice of the initial time $t_{0}$. In cosmology, the quantization of a scalar field is usually performed when the corresponding mode is well inside the horizon, and it is reasonable to choose $t_{0} \rightarrow-\infty$, when the effects of the cosmic expansion are completely negligible. But in any model where the background spacetime is modified from the standard one, the ordinary description of the mode functions becomes valid only after some critical time [12, 17]. In the case of an initially anisotropic universe, we will choose $t_{0}$ to be the time when the universe is isotropized, and the effective theory description becomes valid. We will discuss this in more detail after specifying the interaction terms.

\section{B. Interaction picture}

In calculating $U\left(t, t_{0}\right)$, we decompose $H$ into the (quadratic) kinetic part $H_{0}$ and the interaction part $H_{I}$ that are of higher order in the scalar field amplitudes,

$$
H\left[\phi(t), \pi_{\phi}(t) ; t\right]=H_{0}\left[\phi(t), \pi_{\phi}(t) ; t\right]+H_{I}\left[\phi(t), \pi_{\phi}(t) ; t\right],
$$


where

$$
\begin{aligned}
& H_{0}\left[\phi(t), \pi_{\phi}(t) ; t\right]:=\frac{e^{6 \alpha}}{2} \int d^{3} x\left(e^{-6 \alpha} \pi_{\phi}^{2}+\frac{1}{a^{2}} \phi_{, x}^{2}+\frac{1}{b^{2}} \phi_{, \perp}^{2}\right), \\
& H_{I}\left[\phi(t), \pi_{\phi}(t) ; t\right]:=e^{6 \alpha} \int d^{3} x V .
\end{aligned}
$$

We will calculate $U$ as the power series in $H_{I}$. We introduce the interaction picture and define the interacting operators $\phi^{I}(t)$ and $\pi_{\phi}^{I}(t)$ whose dependence is determined by the quadratic part of the Hamiltonian $H_{0}$,

$$
\dot{\phi}^{I}(t)=i\left[H_{0}\left[\phi^{I}(t), \pi_{\phi}^{I}(t) ; t\right], \phi^{I}(t)\right], \quad \dot{\pi}_{\phi}^{I}(t)=i\left[H_{0}\left[\phi^{I}(t), \pi_{\phi}^{I}(t) ; t\right], \pi_{\phi}^{I}(t)\right]
$$

where the initial conditions are given by $\phi^{I}\left(t_{0}\right)=\phi\left(t_{0}\right)$ and $\pi_{\phi}^{I}\left(t_{0}\right)=\pi_{\phi}\left(t_{0}\right)$.

In evaluating $H_{0}\left[\phi^{I}(t), \pi_{\phi}^{I}(t) ; t\right]$ we can take the time argument of $\phi^{I}$ and $\pi_{\phi}^{I}$ to be any value, and we can take $t_{0}$ so that

$$
H_{0}\left[\phi^{I}(t), \pi_{\phi}^{I}(t) ; t\right]=H_{0}\left[\phi\left(t_{0}\right), \pi_{\phi}\left(t_{0}\right) ; t\right]
$$

The solution to Eq. (10) can be written in terms of the unitality transoformation $\phi^{I}(t)=U_{0}^{-1}\left(t, t_{0}\right) \phi\left(t_{0}\right) U_{0}\left(t, t_{0}\right)$ and $\pi_{\phi}^{I}(t)=U_{0}^{-1}\left(t, t_{0}\right) \pi_{\phi}\left(t_{0}\right) U_{0}\left(t, t_{0}\right)$, where $U_{0}\left(t, t_{0}\right)$ is the solution to the equation

$$
\frac{d}{d t} U_{0}\left(t, t_{0}\right)=-i H_{0}\left[\phi\left(t_{0}\right), \pi_{\phi}\left(t_{0}\right) ; t\right] U_{0}\left(t, t_{0}\right)
$$

We obtain

$$
\frac{d}{d t}\left[U_{0}^{-1}\left(t, t_{0}\right) U\left(t, t_{0}\right)\right]=-i U_{0}^{-1}\left(t, t_{0}\right) H_{I}\left[\phi\left(t_{0}\right), \pi_{\phi}\left(t_{0}\right) ; t\right] U\left(t, t_{0}\right)
$$

which gives

$$
U\left(t, t_{0}\right)=U_{0}\left(t, t_{0}\right) \mathcal{F}\left(t, t_{0}\right), \quad \frac{d}{d t} \mathcal{F}\left(t, t_{0}\right)=-i H_{I}(t) \mathcal{F}\left(t, t_{0}\right), \quad \mathcal{F}\left(t_{0}, t_{0}\right)=1
$$

We define the shorthand notation for the interaction Hamiltonian

$$
H_{I}(t):=U_{0}\left(t, t_{0}\right) H_{I}\left[\phi\left(t_{0}\right), \pi_{\phi}\left(t_{0}\right) ; t\right] U_{0}^{-1}\left(t, t_{0}\right)=H_{I}\left[\phi^{I}(t), \pi_{\phi}^{I}(t) ; t\right] .
$$

The solution for $\mathcal{F}\left(t, t_{0}\right)$ is given by

$$
\mathcal{F}\left(t, t_{0}\right)=T \exp \left(-i \int_{t_{0}}^{t} H_{I}(t) d t\right)
$$

where $T$ is the time-ordering operator. The expectation value for some operator $\mathcal{A}(t)$ is given by

$$
\langle\mathcal{A}(t)\rangle=\left\langle\left[\tilde{T} \exp \left(i \int_{t_{0}}^{t} H_{I}(t) d t\right)\right] \mathcal{A}^{I}(t)\left[T \exp \left(-i \int_{t_{0}}^{t} H_{I}(t) d t\right)\right]\right\rangle
$$

where $\mathcal{A}(t)$ can be any product of $\phi(t) \mathrm{s}$ and $\pi_{\phi}(t) \mathrm{s}$. For the evaluations of the higher order spectra, it is more useful to rewrite Eq. (17) as

$$
\langle\mathcal{A}(t)\rangle=\sum_{N=0}^{\infty} i^{N} \int_{t_{0}}^{t} d t_{N} \int_{t_{0}}^{t_{N}} d t_{N-1} \cdots \int_{t_{0}}^{t_{2}} d t_{1}\left\langle\left[H_{I}\left(t_{1}\right),\left[H_{I}\left(t_{2}\right), \cdots\left[H_{I}\left(t_{N}\right), \mathcal{A}^{I}(t)\right] \cdots\right]\right]\right\rangle
$$

\section{PREINFLATIONARY ANISOTROPIC UNIVERSE AND THE BEHAVIOR OF A SCALAR FIELD}

In this section, we present the background geometry and the way to quantize a scalar field in this background. 


\section{A. Planar Kasner-de Sitter spacetime}

We consider the Einstein gravity with a positive cosmological constant $\Lambda>0$

$$
S_{g}=\frac{1}{2 \kappa^{2}} \int d^{4} x \sqrt{-g}(R-2 \Lambda)
$$

There is the planar Kasner-de Sitter solution with a two-dimensional Euclidean symmetry whose metric is given by Eq. (2) with

$$
a=\sinh ^{\frac{1}{3}}\left(3 H_{0} \tau\right) \tanh ^{\frac{2}{3}}\left(\frac{3}{2} H_{0} \tau\right), \quad b=\sinh ^{\frac{1}{3}}\left(3 H_{0} \tau\right) \operatorname{coth}^{\frac{1}{3}}\left(\frac{3}{2} H_{0} \tau\right)
$$

where $H_{0}=\sqrt{\frac{\Lambda}{3}}$ is the Hubble constant in the isotropized limit. The averaged scale factor is given by

$$
e^{\alpha}:=\left(a b^{2}\right)^{\frac{1}{3}}=\left(\sinh \left(3 H_{0} \tau\right)\right)^{\frac{1}{3}}
$$

The spacetime is completely regular everywhere even in the limit of the intial time $\tau \rightarrow 0$, in contrast to the other branches of the Kasner spacetime. The Hubble parameter in each direction is given by

$$
H_{a}:=\frac{\dot{a}}{a}=\frac{H_{0}}{\sinh \left(3 H_{0} \tau\right)}\left(2+\cosh \left(3 H_{0} \tau\right)\right), \quad H_{b}:=\frac{\dot{b}}{b}=H_{0} \tanh \left(\frac{3}{2} H_{0} \tau\right) .
$$

In this solution, at the initial times the spacetime structure becomes the product of a (Milne) patch of the twodimensional Minkowski spacetime and the two-dimensional Euclidean space. Thus it is possible to define the initial adianatic vacuum state in this solution. At the later times for $\tau \rightarrow \infty$, the universe approaches the de Sitter spacetime with the expansion $H_{0}$. In the other Kasner-de Sitter solutions which contain initial curvature singularities, the adiabatic vacuum cannot be well-defined in the asymptotic past [10, 11].

\section{B. Scalar field in the Kasner-de Sitter spacetime}

Here as in Sec. II, we will work in the time coordinate $d t=\frac{d \tau}{e^{3 \alpha}}$. Note that these two time coordinates are related via the simple analytic relation

$$
\sinh \left(-3 H_{0} t\right)=\frac{1}{\sinh \left(3 H_{0} \tau\right)}
$$

In the interaction picture,

$$
\phi^{I}=\int \frac{d^{3} k}{(2 \pi)^{\frac{3}{2}}} e^{i \mathbf{k x}} \phi(t, \mathbf{k})=\int \frac{d^{3} k}{(2 \pi)^{\frac{3}{2}}} e^{i \mathbf{k x}}\left(u_{\mathbf{k}}(t) a_{\mathbf{k}}+u_{-\mathbf{k}}^{*}(t) a_{-\mathbf{k}}^{\dagger}\right) .
$$

In our Kasner-de Sitter background,

$$
\left(\frac{d^{2}}{d t^{2}}+\Omega(t)^{2}\right) u_{\mathbf{k}}(t)=0, \quad \Omega^{2}(t):=\frac{2^{4 / 3}\left(k_{\perp}^{2} e^{6 H t}+k_{1}^{2}\right)}{\left(1-e^{6 H t}\right)^{4 / 3}}=\frac{2^{4 / 3} k^{2}}{\xi^{4 / 3}(t)}\left(1-r_{\perp}^{2} \xi(t)\right)
$$

where $k^{2}=k_{1}^{2}+k_{\perp}^{2}, r_{\perp}:=k_{\perp} / k$ and

$$
\xi(t):=1-e^{6 H t}=e^{-6 \alpha}\left(\sqrt{e^{6 \alpha}+1}-1\right),
$$

varies from one to zero as time $t$ increases from negative infinity to zero. The late time solution is given by

$$
u_{\mathbf{k}}(t)=A_{\mathbf{k}}^{+} u_{\mathbf{k}}^{(0)}(t)+A_{\mathbf{k}}^{-} u_{\mathbf{k}}^{(0) *}(t), \quad u_{\mathbf{k}}^{(0)}(t)=\frac{e^{i \frac{k}{H_{0}}\left(-3 H_{0} t\right)^{\frac{1}{3}}}}{\sqrt{2 k}}\left[\left(-3 H_{0} t\right)^{\frac{1}{3}}+\frac{i H_{0}}{k}\right] .
$$

The coefficients $A_{\mathbf{k}}^{+}$and $A_{\mathbf{k}}^{-}$are determined through the matching of the mode functions. In Refs. [10, 11], we have classified modes into the nonplanar high-momentum modes and the planar ones. 


\section{(1) Nonplanar, high-momentum modes}

The nonplanar high-momentum modes satisfy $H_{0} \ll k_{1} \sim k_{\perp}$. For these modes, the WKB solutions for the early time can be directly matched to mode functions in the de Sitter spacetime at the later times. The coefficients can be found as

$$
A_{\mathrm{k}}^{+}=\left(1-\frac{H_{0}}{2 k}\right)\left(1-i \sqrt{\frac{H_{0}}{k}}\right), \quad A_{\mathrm{k}}^{-}=\frac{1}{2}\left(\frac{H_{0}}{k}\right)^{\frac{3}{2}} Q\left(r_{\perp}\right),
$$

where $Q\left(r_{\perp}\right):=\frac{2}{3}-r_{\perp}^{2}$ contains the direction dependence appearing only in the negative frequency mode. $A_{\mathbf{k}}^{(-)}$is of order $\epsilon_{*}^{3}$ where $\epsilon_{*}$ is the adiabaticity parameter $\epsilon:=\frac{\left(\Omega^{2}\right)_{,}}{2 \Omega^{3}}$ at the matching time $t=t_{*}$. Hence, we expect that these modes would not give significant effects on spectra and cosmic observables at the level of the power spectrum.

(2) Planar modes

We then consider the planar modes with $H_{0}<k_{1} \ll k_{\perp}$, which are along the constant $x$ plane where there is a two-dimensional rotational symmetry. The main difference from the previous case is that there is a temporal violation of the WKB approximation during which effects of the anisotropy are encoded into the mode functions. According to Refs [10, 11],

$$
A_{\mathbf{k}}^{+}=\frac{1}{\left(1-e^{-2 \pi q_{1}}\right)^{\frac{1}{2}}} e^{-i \sqrt{\frac{k}{H_{0}}}-i \Phi+i\left(\frac{\pi}{4}-\sqrt{\frac{k_{1}}{H_{0}}}\right)}, \quad A_{\mathbf{k}}^{-}=\frac{e^{-\pi q_{1}}}{\left(1-e^{-2 \pi q_{1}}\right)^{\frac{1}{2}}} e^{i \sqrt{\frac{k}{H_{0}}}+i \Phi-i\left(\frac{\pi}{4}-\sqrt{\frac{k_{1}}{H_{0}}}\right)},
$$

where $q_{1}:=\frac{2^{\frac{2}{3}} k_{1}}{3 H_{0}}$ and $\Phi:=\frac{\sqrt{\pi} \Gamma\left(\frac{1}{3}\right) k}{3 \times 2^{\frac{1}{3}} \Gamma\left(\frac{5}{6}\right) H_{0}}+O\left(\sqrt{\frac{k}{H_{0}}}\right)$. Since $e^{-\pi q_{1}}$ may be close to unity, $\left|A_{\mathbf{k}}^{(-)}\right| \lesssim\left|A_{\mathbf{k}}^{(+)}\right|$.

To discuss the late time behavior of the mode functions, it is convenient to work by choosing the conformal time $d \eta=e^{-\alpha} d \tau=e^{2 \alpha} d t$, in which the late time mode functions can be rewritten as

$$
u_{\mathbf{k}}(\eta)=A_{\mathbf{k}}^{+} u_{\mathbf{k}}^{(0)}(\eta)+A_{\mathbf{k}}^{-} u_{\mathbf{k}}^{(0) *}(\eta), \quad u_{\mathbf{k}}^{(0)}(\eta)=\frac{H_{0} e^{-i k \eta}}{\sqrt{2 k}}\left(-\eta+\frac{i}{k}\right)
$$

In the conformal time coordinate, the averaged scale factor is given by $e^{\alpha}=-\frac{1}{H_{0} \eta}$.

\section{Interaction Hamiltonian}

In this subsection, we focus on the choice of $V$ in Eq. (1). We consider the high energy corrections to the kinetic terms. Although in general as such corrections there would be both the time- and spatial-derivative terms, for simplicity we focus on the time-derivative terms. The time derivatives of the scalar field in the Lagrangian density except for the volume factor can be expanded in terms of the amplitude

$$
\frac{1}{2} \phi_{, \tau}^{2}+\sum_{n=3} q_{n} \frac{\phi_{, \tau}^{n}}{M^{2(n-2)}}
$$

where $q_{n}(n=3,4, \cdots)$ are dimensionless constants which are typically of order unity, and $M$ is the energy scale at which the higher order corrections become important. It is also appropriate to use derivatives with respect to the proper time $\tau$, rather than another time coordinate, such as $t$. We may regard our scalar field theory as the low energy limit of a complete UV theory. appear just as low energy corrections. In the interaction picture, the leading order quadratic term gives the ordinary kinetic term, and higher order ones give interactions. Since we are particularly interested in the three- and four-point interactions, we focus on the terms of $n=3$ and $n=4$. We expect that the time-derivative interactions would give us information enough to see at least the difference of the anisotropic background from the de Sitter inflation.

Thus we choose $V$ in Eq. (11) as

$$
V=e^{-6 \alpha} \pi_{\phi}^{2}\left[q_{3} e^{-3 \alpha} \frac{\pi_{\phi}}{M^{2}}+q_{4} e^{-6 \alpha}\left(\frac{\pi_{\phi}}{M^{2}}\right)^{2}\right] .
$$

Using $\pi_{\phi}=\dot{\phi}$ and $d t=e^{-3 \alpha} d \tau$, Eq. (32) is rewritten as

$$
V=\phi_{, \tau}^{2}\left[q_{3} \frac{\phi_{, \tau}}{M^{2}}+q_{4}\left(\frac{\phi_{, \tau}}{M^{2}}\right)^{2}\right]
$$


which agrees with the $n=3$ and $n=4$ terms in Eq. (31). From Eq. (99), the interaction Hamiltonian is given by

$$
\begin{aligned}
H_{I}[\phi(t), \pi(t) ; t] & =H_{I}^{(3)}[\phi(t), \pi(t) ; t]+H_{I}^{(4)}[\phi(t), \pi(t) ; t] \\
& =e^{6 \alpha} \int d^{3} x \phi_{, \tau}^{2}\left[q_{3} \frac{\phi_{, \tau}}{M^{2}}+q_{4}\left(\frac{\phi_{, \tau}}{M^{2}}\right)^{2}\right], \\
H_{I}^{(3)}[\phi(t), \pi(t) ; t] & :=e^{6 \alpha} \int d^{3} x \frac{q_{3}}{M^{2}} \phi_{, \tau}^{3}=e^{-3 \alpha} \int d^{3} x \frac{q_{3}}{M^{2}} \dot{\phi}^{3}, \\
H_{I}^{(4)}[\phi(t), \pi(t) ; t] & :=e^{6 \alpha} \int d^{3} x \frac{q_{4}}{M^{4}} \phi_{, \tau}^{4}=e^{-6 \alpha} \int d^{3} x \frac{q_{4}}{M^{4}} \dot{\phi}^{4} .
\end{aligned}
$$

Let us focus more on the reasons for considering the derivative interactions. The first reason is that in the metric perturbation theory (most of) the interactions are given by the interactions of derivatives 14]. The second reason is that higher derivative interactions may enhance the higher order spectra [17, 20] because the interactions depend on the inverse power of the (averaged) scale factor, which is large in the past. Although we ignore the backreaction of the scalar field on the geometry, our analysis should contain overlap with the realistic analysis on the quantum fluctuations. Since $\left\langle\left|\phi_{, \tau}\right|^{2}\right\rangle \simeq H_{0}^{4}$, the scalar field does not backreact significantly on the background geometry so long as $H_{0}<M_{p}$. In addition, in the equation of motion of the scalar field the corrections of the higher order derivative terms to the kinetic term appear in order of $\frac{H_{0}^{2}}{M^{2}}$. Thus, the higher-order derivative interactions can always be treated as perturbations so long as $M>H_{0}$, and then the early time WKB solutions and late time de Sitter ones given by Eq. (27) are good approximations of the mode functions with a very high accuracy. Hence, our perturbative arguments are valid when the relation $H_{0}<M \lesssim M_{p}$ is satisfied. We choose the cut-off time for the time integral to be $\frac{k}{e^{\alpha}}=M$ when the physical momentum for the corresponding mode becomes below the energy scale of the derivative interactions. This gives $-\eta_{0}=\frac{M}{k H_{0}}$, where $k$ is the total momentum for the relevant spectra, i.e. $k=k_{1}+k_{2}+k_{3}$ for the bispectra and $k=k_{1}+k_{2}+k_{3}+k_{4}$ for the trispectra. This time becomes later than the time when the de Sitter mode function becomes valid, $-\eta_{0}<-\eta_{*}:=\frac{1}{\sqrt{k H_{0}}}$, leading to $k<\frac{M^{2}}{H_{0}}$. In other words, our interest is in the energy scale satisfying

$$
\frac{M}{H_{0}}<\epsilon_{*}^{-1},
$$

for a corresponding number of $k$. Thus, in the subsequent parts of this paper we will focus on these modes.

In the next sections, we will present the bispectra and trispectra following the formulation presented in the previous section. The leading order contribution to the bispectrum is given by the three-point contact interaction diagram. Similarly, the leading order contribution to the trispectra is given by two kinds of the connected diagrams. The first one is the contact interaction diagram [see, e.g., Fig. 1 (A) in [19]], and the second one is the scalar exchange interaction diagram [see, e.g., Fig. 1 (B) in [19]]. We expect that loop corrections are suppressed and ignore them in the subsequent discussions.

\section{Power spectra}

Before closing this section, we show the power spectra including the leading order corrections due to the primordial anisotropy, for both the nonplanar high-momentum modes and the planar modes, which were obtained in [10, 11]. Note that, in contrast to the power spectra obtained from the standard isotropic scenario, they depend not only on the magnitude of the momentum vector $k=|\mathbf{k}|$, but also on its direction. For the nonplanar high-momentum modes, the power spectrum is given by

$$
P(\mathbf{k})=\left(\frac{H_{0}}{2 \pi}\right)^{2}\left(1+Q\left(r_{\perp}\right)\left(\frac{H_{0}}{k}\right)^{3 / 2} \cos \left(2 \sqrt{\frac{k}{H_{0}}}\right)\right),
$$

where $Q\left(r_{\perp}\right)$ is defined below Eq. (28). Thus the corrections due to the primordial anisotropy are suppressed by $\epsilon_{*}^{3}$.

On the other hand, for the planar modes, the power spectrum is given by

$$
P(\mathbf{k})=\left(\frac{H_{0}}{2 \pi}\right)^{2}\left(\operatorname{coth}\left(\pi q_{1}\right)-\frac{\sin (2 \Phi)}{\sinh \left(\pi q_{1}\right)}\right),
$$

where $q_{1}$ and $\Phi$ are defined below Eq. (29). Thus the corrections due to the primordial anisotropy are not suppressed. 


\section{BISPECTRA}

The leading order contribution to the bispectra is given by the contact interaction

$$
\left\langle\phi\left(t, \mathbf{k}_{1}\right) \phi\left(t, \mathbf{k}_{2}\right) \phi\left(t, \mathbf{k}_{3}\right)\right\rangle=i \int_{t_{0}}^{t} d t_{1}\left\langle\left[H_{I}^{(3)}\left(t_{1}\right), \phi^{I}\left(t, \mathbf{k}_{1}\right) \phi^{I}\left(t, \mathbf{k}_{2}\right) \phi^{I}\left(t, \mathbf{k}_{3}\right)\right]\right\rangle .
$$

The bispectrum in the $t \rightarrow 0$ limit takes the form of

$$
\left\langle\phi\left(0, \mathbf{k}_{1}\right) \phi\left(0, \mathbf{k}_{2}\right) \phi\left(0, \mathbf{k}_{3}\right)\right\rangle=(2 \pi)^{-3} \frac{q_{3}}{M^{2}} B\left(\mathbf{k}_{1}, \mathbf{k}_{2}, \mathbf{k}_{3}\right) \delta\left(\mathbf{k}_{1}+\mathbf{k}_{2}+\mathbf{k}_{3}\right) .
$$

After computations, we obtain

$$
\begin{aligned}
& B\left(\mathbf{k}_{1}, \mathbf{k}_{2}, \mathbf{k}_{3}\right) \\
= & -\frac{3 H_{0}^{5}}{4\left(k_{1} k_{2} k_{3}\right)}\left\{i\left(A_{\mathbf{k}_{1}}^{+}-A_{\mathbf{k}_{1}}^{-}\right)^{*}\left(A_{\mathbf{k}_{2}}^{+}-A_{\mathbf{k}_{2}}^{-}\right)^{*}\left(A_{\mathbf{k}_{3}}^{+}-A_{\mathbf{k}_{3}}^{-}\right)^{*}\right. \\
\times & {\left[-A_{\mathbf{k}_{1}}^{+} A_{\mathbf{k}_{2}}^{+} A_{\mathbf{k}_{3}}^{+} \mathcal{I}\left(k_{1}, k_{2}, k_{3}\right)+A_{\mathbf{k}_{1}}^{-} A_{\mathbf{k}_{2}}^{-} A_{\mathbf{k}_{3}}^{-} \mathcal{I}\left(-k_{1},-k_{2},-k_{3}\right)\right.} \\
+ & \left.\left(A_{\mathbf{k}_{1}}^{+} A_{\mathbf{k}_{2}}^{+} A_{\mathbf{k}_{3}}^{-} \mathcal{I}\left(k_{1}, k_{2},-k_{3}\right)+2 \text { perms }\right)-\left(A_{\mathbf{k}_{1}}^{+} A_{\mathbf{k}_{2}}^{-} A_{\mathbf{k}_{3}}^{-} \mathcal{I}\left(k_{1},-k_{2},-k_{3}\right)+2 \text { perms }\right)\right] \\
+ & C . C .\}
\end{aligned}
$$

where

$$
\begin{aligned}
& \mathcal{I}\left(p_{1}, p_{2}, p_{3}\right) \\
:= & \frac{1}{\left(p_{1}+p_{2}+p_{3}\right)^{3}}\left\{2 i-e^{-i\left(p_{1}+p_{2}+p_{3}\right) \eta_{0}}\left[2 i-2\left(p_{1}+p_{2}+p_{3}\right) \eta_{0}-i\left(p_{1}+p_{2}+p_{3}\right)^{2} \eta_{0}^{2}\right]\right\},
\end{aligned}
$$

and $\mathcal{I}\left(p_{1}, p_{2}, p_{3}\right)^{*}=\mathcal{I}\left(-p_{1},-p_{2},-p_{3}\right)$. In the limit of $p_{1}+p_{2}+p_{3} \rightarrow 0$, the function $\mathcal{I}$ remains finite,

$$
\mathcal{I}\left(p_{1}, p_{2}, p_{3}\right)=\frac{\eta_{0}^{3}}{3}+O\left(p_{1}+p_{2}+p_{3}\right)
$$

Since $\left|\mathcal{I}\left(p_{1}, p_{2}, p_{3}\right)\right|$ is increasing in the limit of $p_{1}+p_{2}+p_{3} \rightarrow 0$, the bispectrum would have peaks in the limits of $k_{1} \rightarrow k_{2}+k_{3}, k_{2} \rightarrow k_{1}+k_{3}$ and $k_{3} \rightarrow k_{1}+k_{2}$ (case I). The bispectrum can also have a peak in the limits $k_{1}=k_{2} \gg k_{3}$, $k_{2}=k_{3} \gg k_{1}$, and $k_{3}=k_{1} \gg k_{2}$ (case II). We discuss each case separately.

\section{Case $I$}

In the limit of of $k_{1} \rightarrow k_{2}+k_{3}$, the leading order contribution to the bispectrum is given by

$$
\begin{aligned}
& B\left(\mathbf{k}_{1}, \mathbf{k}_{2}, \mathbf{k}_{3}\right) \\
\rightarrow & \frac{H_{0}^{5} \eta_{0}^{3}}{2\left(k_{1} k_{2} k_{3}\right)} \operatorname{Im}\left\{\left(A_{\mathbf{k}_{1}}^{+}-A_{\mathbf{k}_{1}}^{-}\right)^{*}\left(A_{\mathbf{k}_{2}}^{+}-A_{\mathbf{k}_{2}}^{-}\right)^{*}\left(A_{\mathbf{k}_{3}}^{+}-A_{\mathbf{k}_{3}}^{-}\right)^{*}\left(A_{\mathbf{k}_{1}}^{-} A_{\mathbf{k}_{2}}^{+} A_{\mathbf{k}_{3}}^{+}-A_{\mathbf{k}_{1}}^{+} A_{\mathbf{k}_{2}}^{-} A_{\mathbf{k}_{3}}^{-}\right)\right\} .
\end{aligned}
$$

Similar results are obtained in the limits of $k_{2} \rightarrow k_{1}+k_{3}$ and $k_{3} \rightarrow k_{1}+k_{2}$. For the bispectrum for the nonplanar high-momentum modes,

$$
\operatorname{Im}\left\{\left(A_{\mathbf{k}_{1}}^{+}-A_{\mathbf{k}_{1}}^{-}\right)^{*}\left(A_{\mathbf{k}_{2}}^{+}-A_{\mathbf{k}_{2}}^{-}\right)^{*}\left(A_{\mathbf{k}_{3}}^{+}-A_{\mathbf{k}_{3}}^{-}\right)^{*}\left(A_{\mathbf{k}_{1}}^{-} A_{\mathbf{k}_{2}}^{+} A_{\mathbf{k}_{3}}^{+}-A_{\mathbf{k}_{1}}^{+} A_{\mathbf{k}_{2}}^{-} A_{\mathbf{k}_{3}}^{-}\right)\right\} \simeq \frac{Q\left(r_{\perp, 1}\right)}{2} \frac{H_{0}^{2}}{k_{1}^{2}}
$$

Thus

$$
B\left(\mathbf{k}_{1}, \mathbf{k}_{2}, \mathbf{k}_{3}\right) \rightarrow \frac{H_{0}^{7} \eta_{0}^{3}}{4\left(k_{1}^{3} k_{2} k_{3}\right)} Q\left(r_{\perp, 1}\right)
$$


The ratio of Eq. (45) to the case of the de Sitter inflation, Eq. (A1), is given by

$$
Q\left(r_{\perp, 1}\right) H_{0}^{2} k_{1} \eta_{0}^{3}=Q\left(r_{\perp, 1}\right) \frac{M^{3}}{H_{0}^{3}} \frac{k_{1} H_{0}^{2}}{k^{3}}
$$

which is of order $\frac{M^{3}}{H_{0}^{3}} \epsilon_{*}^{4}$. Thus the direction dependent bispectrum can be as large as that in the de Sitter inflation for $\frac{M}{H_{0}}>\epsilon_{*}^{-\frac{4}{3}}$. But this is never satisfied because of Eq. (35).

On the other hand, for the planar modes, using the phase factor $\psi:=-\sqrt{\frac{k}{H_{0}}}-\Phi+\left(\frac{\pi}{4}-\sqrt{\frac{k_{1}}{H_{0}}}\right)$ the bispectrum in the same limit is given by

$$
B\left(\mathbf{k}_{1}, \mathbf{k}_{2}, \mathbf{k}_{3}\right) \rightarrow-\frac{H_{0}^{5} \eta_{0}^{3}}{2\left(k_{1} k_{2} k_{3}\right)} \frac{e^{-\pi q_{1,1}} \sin \left(2 \psi_{1}\right)}{\left(1-e^{-2 \pi q_{1,1}}\right)\left(1-e^{-2 \pi q_{1,2}}\right)\left(1-e^{-2 \pi q_{1,3}}\right)} .
$$

The ratio to the case of the de Sitter inflation is given by

$$
\frac{M^{3}}{H_{0}^{3}} \frac{k_{1}^{3}}{k^{3}} \frac{e^{-\pi q_{1,1}} \sin \left(2 \psi_{1}\right)}{\left(1-e^{-2 \pi q_{1,1}}\right)\left(1-e^{-2 \pi q_{1,2}}\right)\left(1-e^{-2 \pi q_{1,3}}\right)}
$$

which is greater than unity since $\frac{M}{H_{0}} \gg 1$. This bound is consistent with Eq. (35) for $1<\frac{M}{H_{0}}<\epsilon_{*}^{-1}$. Therefore, such a bispectrum is more important than for the case of the nonplanar high-momentum modes. This bound can be consistent with Eq. (35).

Similar results can be obtained for the limits of $k_{2} \rightarrow k_{3}+k_{1}$, and $k_{3} \rightarrow k_{1}+k_{2}$.

\section{Case II}

In the limit of $k_{1}=k_{2} \gg k_{3}$, the leading order contribution to the bispectrum from the nonplanar high-momentum modes is given by

$$
\begin{aligned}
B\left(\mathbf{k}_{1}, \mathbf{k}_{2}, \mathbf{k}_{3}\right) & \rightarrow \frac{H_{0}^{5} \eta_{0}^{3}}{2\left(k_{1} k_{2} k_{3}\right)} \operatorname{Im}\left\{\left(A_{\mathbf{k}_{1}}^{+}-A_{\mathbf{k}_{1}}^{-}\right)^{*}\left(A_{\mathbf{k}_{2}}^{+}-A_{\mathbf{k}_{2}}^{-}\right)^{*}\left|A_{\mathbf{k}_{3}}^{+}-A_{\mathbf{k}_{3}}^{-}\right|^{2}\left(A_{\mathbf{k}_{1}}^{+} A_{\mathbf{k}_{2}}^{-}+A_{\mathbf{k}_{1}}^{-} A_{\mathbf{k}_{2}}^{+}\right)\right\} \\
& \simeq \frac{H_{0}^{7} \eta_{0}^{3}}{4 k_{1}^{4} k_{3}}\left(Q\left(r_{\perp, 1}\right)+Q\left(r_{\perp, 2}\right)\right) .
\end{aligned}
$$

The ratio of Eq. (49) to the case of the de Sitter inflation, Eq. (A1), is given by

$$
k_{1} H_{0}^{2} \eta_{0}^{3}\left(Q\left(r_{\perp, 1}\right)+Q\left(r_{\perp, 2}\right)\right)=\frac{M^{3}}{H_{0}^{3}} \frac{k_{1} H_{0}^{2}}{k^{3}}\left(Q\left(r_{\perp, 1}\right)+Q\left(r_{\perp, 2}\right)\right),
$$

which is of order $\frac{M^{3}}{H_{0}^{3}} \epsilon_{*}^{4}$. Thus the direction dependent bispectrum can be as large as that in the de Sitter inflation for $\frac{M}{H_{0}}>\epsilon_{*}^{-\frac{4}{3}}$. But this is never satisfied because of Eq. (35). In addition, assuming that $k=k_{1}+k_{2}+k_{3}$ is fixed, the ratio of the bispectrum of case II, Eq. (49), to that of case I, Eq. (45) (with the permutation to $k_{3} \rightarrow k_{1}+k_{2}$ ) is given by $\frac{k}{k_{3}}$. Thus the bispectrum in case II is enhanced to that in case I by the ratio $\frac{k}{k_{3}} \gg 1$.

The leading order contribution from the planar modes is given by

$$
B\left(\mathbf{k}_{1}, \mathbf{k}_{2}, \mathbf{k}_{3}\right) \simeq-\frac{H_{0}^{5} \eta_{0}^{3}}{2\left(k_{1}^{2} k_{3}\right)} \frac{e^{-\pi q_{1,1}} \sin \left(2 \psi_{1}\right)+e^{-\pi q_{1,2}} \sin \left(2 \psi_{2}\right)}{\left(1-e^{-2 \pi q_{1,1}}\right)\left(1-e^{-2 \pi q_{1,2}}\right)\left(1-e^{-2 \pi q_{1,3}}\right)} .
$$

The ratio to the case of the de Sitter inflation is given by

$$
\frac{M^{3}}{H_{0}^{3}} \frac{k_{1}^{3}}{k^{3}} \frac{e^{-\pi q_{1,1}} \sin \left(2 \psi_{1}\right)+e^{-\pi q_{1,2}} \sin \left(2 \psi_{2}\right)}{\left(1-e^{-2 \pi q_{1,1}}\right)\left(1-e^{-2 \pi q_{1,2}}\right)\left(1-e^{-2 \pi q_{1,3}}\right)},
$$

which is greater than unity since $\frac{M}{H_{0}} \gg 1$. This bound is consistent with Eq. (35) for $1<\frac{M}{H_{0}}<\epsilon_{*}^{-1}$. The ratio of Eq. (47) with permutation to $k_{3} \rightarrow k_{1}+k_{2}$ to Eq. (51) is given by

$$
\frac{k_{3}}{k} \frac{e^{-\pi q_{1,3}} \sin \left(2 \psi_{3}\right)}{e^{-\pi q_{1,1}} \sin \left(2 \psi_{1}\right)+e^{-\pi q_{1,2}} \sin \left(2 \psi_{2}\right)},
$$

which becomes smaller than unity as for the nonplanar high-momentum modes.

A similar result can be obtained for the cases of $k_{2}=k_{3} \gg k_{1}$ and $k_{3}=k_{1} \gg k_{2}$. 


\section{Features of the bispectra}

We summarize the features of the bispectra in our background spacetime and compare them with those in the de Sitter inflation. In our background the bispectrum in the limit of case I (for example, for $k_{1} \rightarrow k_{2}+k_{3}$ ) is greater than that in the limit of case II (for example, for $k_{1}=k_{2} \gg k_{3}$ ), by a factor of $\frac{k}{k_{3}}$, where $k$ is the typical magnitude of the momentum vectors.

We then discuss the direction dependences of the bispectra. Though we focus on the nonplanar high-momentum modes, the essence is the same also for the planar modes. In case I, for example, for $k_{1} \rightarrow k_{2}+k_{3}$, the bispectrum is proportional to $Q\left(r_{\perp, 1}\right)$ and hence depends on the direction of the momentum vector $\mathbf{k}_{1}$. In case II, for example, for $k_{1}=k_{2} \gg k_{3}$, it is proportional to $Q\left(r_{\perp, 1}\right)+Q\left(r_{\perp, 2}\right)$, and hence depends on the directions of the momenta $\mathbf{k}_{1}$ and $\mathbf{k}_{2}$, which have shorter wavelengths than $\mathbf{k}_{3}$. Thus the bispectra are direction dependent, and the dependence is significantly different in various limiting cases.

We finally compare the amplitude of the bispectra with those in the de Sitter inflation. For the planar modes, the bispectra in both the limits of cases I and II can be greater than those in the de Sitter spacetime. On the other hand, for the nonplanar high-momentum modes, the bispectrum in both cases cannot exceed those in the de Sitter inflation. Thus, combined with the direction dependence, the bispectra for the planar modes in all the limiting cases would be particularly interesting to distinguish models.

In the next section, we will investigate the trispectra. In contrast to the bispectra, even for the nonplanar highmomentum modes, the trispectra could be more important than those in the de Sitter inflation.

\section{TRISPECTRA}

The leading order contributions to the trispectra are given by the contact interaction as well as the scalar exchange interaction.

\section{A. Contribution from the contact interaction}

The leading order contribution of the four-point interaction is given by

$$
\left\langle\phi\left(t, \mathbf{k}_{1}\right) \phi\left(t, \mathbf{k}_{2}\right) \phi\left(t, \mathbf{k}_{3}\right) \phi\left(t, \mathbf{k}_{4}\right)\right\rangle=i \int_{t_{0}}^{t} d t_{1}\left\langle\left[H_{I}^{(4)}\left(t_{1}\right), \phi^{I}\left(t, \mathbf{k}_{1}\right) \phi^{I}\left(t, \mathbf{k}_{2}\right) \phi^{I}\left(t, \mathbf{k}_{3}\right) \phi^{I}\left(t, \mathbf{k}_{4}\right)\right]\right\rangle .
$$

The trispectrum in the $t \rightarrow 0$ limit takes the following form:

$$
\left\langle\phi\left(0, \mathbf{k}_{1}\right) \phi\left(0, \mathbf{k}_{2}\right) \phi\left(0, \mathbf{k}_{3}\right) \phi\left(0, \mathbf{k}_{4}\right)\right\rangle=(2 \pi)^{-3} \frac{q_{4}}{M^{4}} T_{c}\left(\mathbf{k}_{1}, \mathbf{k}_{2}, \mathbf{k}_{3}, \mathbf{k}_{4}\right) \delta\left(\mathbf{k}_{1}+\mathbf{k}_{2}+\mathbf{k}_{3}+\mathbf{k}_{4}\right) .
$$

After computations, we obtain

$$
\begin{aligned}
T_{c}\left(\mathbf{k}_{1}, \mathbf{k}_{2}, \mathbf{k}_{3}, \mathbf{k}_{4}\right) & =\frac{6 i H_{0}^{8}}{\left(k_{1} k_{2} k_{3} k_{4}\right)}\left[\left(A_{\mathbf{k}_{1}}^{+}-A_{\mathbf{k}_{1}}^{-}\right)^{*}\left(A_{\mathbf{k}_{2}}^{+}-A_{\mathbf{k}_{2}}^{-}\right)^{*}\left(A_{\mathbf{k}_{3}}^{+}-A_{\mathbf{k}_{3}}^{-}\right)^{*}\left(A_{\mathbf{k}_{4}}^{+}-A_{\mathbf{k}_{4}}^{-}\right)^{*}\right. \\
& \times\left\{\left[-A_{\mathbf{k}_{1}}^{+} A_{\mathbf{k}_{2}}^{+} A_{\mathbf{k}_{3}}^{+} A_{\mathbf{k}_{4}}^{+} \mathcal{J}\left(k_{1}, k_{2}, k_{3}, k_{4}\right)-A_{\mathbf{k}_{1}}^{-} A_{\mathbf{k}_{2}}^{-} A_{\mathbf{k}_{3}}^{-} A_{\mathbf{k}_{4}}^{-} \mathcal{J}\left(-k_{1},-k_{2},-k_{3},-k_{4}\right)\right.\right. \\
& +\left(A_{\mathbf{k}_{1}}^{+} A_{\mathbf{k}_{2}}^{+} A_{\mathbf{k}_{3}}^{+} A_{\mathbf{k}_{4}}^{-} \mathcal{J}\left(k_{1}, k_{2}, k_{3},-k_{4}\right)+3 \text { perms }\right) \\
& -\left(A_{\mathbf{k}_{1}}^{+} A_{\mathbf{k}_{2}}^{+} A_{\mathbf{k}_{3}}^{-} A_{\mathbf{k}_{4}}^{-} \mathcal{J}\left(k_{1}, k_{2},-k_{3},-k_{4}\right)+5 \text { perms }\right) \\
& \left.+\left(A_{\mathbf{k}_{1}}^{+} A_{\mathbf{k}_{2}}^{-} A_{\mathbf{k}_{3}}^{-} A_{\mathbf{k}_{4}}^{-} \mathcal{J}\left(k_{1},-k_{2},-k_{3},-k_{4}\right)+3 \text { perms }\right)\right\} \\
& - \text { C.C. }]
\end{aligned}
$$


where we have defined

$$
\begin{aligned}
& \mathcal{J}\left(p_{1}, p_{2}, p_{3}, p_{4}\right) \\
:= & \frac{1}{4\left(p_{1}+p_{2}+p_{3}+p_{4}\right)^{5}}\left\{-24 i+e^{-i\left(p_{1}+p_{2}+p_{3}+p_{4}\right) \eta_{0}}\left[24 i-\left(p_{1}+p_{2}+p_{3}+p_{4}\right) \eta_{0}\right.\right. \\
\times & \left(24-i\left(p_{1}+p_{2}+p_{3}+p_{4}\right) \eta_{0}\left(-12-\left(p_{1}+p_{2}+p_{3}+p_{4}\right) \eta_{0}\right.\right. \\
\times & \left.\left.\left.\left.\left(4 i-\left(p_{1}+p_{2}+p_{3}+p_{4}\right) \eta_{0}\right)\right)\right)\right]\right\} .
\end{aligned}
$$

In the limit of $p_{1}+p_{2}+p_{3}+p_{4} \rightarrow 0$, the function $\mathcal{J}$ remains finite as

$$
\mathcal{J}\left(p_{1}, p_{2}, p_{3}, p_{4}\right)=\frac{1}{20} \eta_{0}^{5}+O\left(p_{1}+p_{2}+p_{3}+p_{4}\right) .
$$

Thus the trispectrum from the contact interaction depends on the size of four vectors $k_{i}(i=1,2,3,4)$, and not on the size of two independent relative vectors $k_{12}=\left|\mathbf{k}_{1}+\mathbf{k}_{2}\right|$ and $k_{14}=\left|\mathbf{k}_{1}+\mathbf{k}_{4}\right|$, since there is no internal line in the diagram. Since $\left|\mathcal{J}\left(p_{1}, p_{2}, p_{3}, p_{4}\right)\right|$ is increasing in the limit of $p_{1}+p_{2}+p_{3}+p_{4} \rightarrow 0$, the trispectrum has the maximum amplitude in the limits of $k_{1} \rightarrow k_{2}+k_{3}+k_{4}, k_{2} \rightarrow k_{1}+k_{3}+k_{4}, k_{3} \rightarrow k_{1}+k_{2}+k_{4}$, and $k_{4} \rightarrow k_{1}+k_{2}+k_{3}$ (case I), as well as $k_{1}+k_{2} \rightarrow k_{3}+k_{4}$, and $k_{1}+k_{4} \rightarrow k_{2}+k_{3}$ (case II). We also discuss the limits of $k_{1}=k_{2} \gg k_{12}$ and $k_{3}=k_{4} \gg k_{12}$, and similarly $k_{1}=k_{4} \gg k_{14}$ and $k_{2}=k_{3} \gg k_{14}$ (case III).

\section{Case $I$}

In the limit of $k_{1} \rightarrow k_{2}+k_{3}+k_{4}$, the leading order contribution to the trispectrum is given by

$$
\begin{aligned}
T_{c}\left(\mathbf{k}_{1}, \mathbf{k}_{2}, \mathbf{k}_{3}, \mathbf{k}_{4}\right) & \rightarrow-\frac{3 H_{0}^{8} \eta_{0}^{5}}{5\left(k_{1} k_{2} k_{3} k_{4}\right)} \operatorname{Im}\left\{\left(A_{\mathbf{k}_{1}}^{+}-A_{\mathbf{k}_{1}}^{-}\right)^{*}\left(A_{\mathbf{k}_{2}}^{+}-A_{\mathbf{k}_{2}}^{-}\right)^{*}\left(A_{\mathbf{k}_{3}}^{+}-A_{\mathbf{k}_{3}}^{-}\right)^{*}\left(A_{\mathbf{k}_{4}}^{+}-A_{\mathbf{k}_{4}}^{-}\right)^{*}\right. \\
& \left.\times\left(A_{\mathbf{k}_{1}}^{-} A_{\mathbf{k}_{2}}^{+} A_{\mathbf{k}_{3}}^{+} A_{\mathbf{k}_{4}}^{+}+A_{\mathbf{k}_{1}}^{+} A_{\mathbf{k}_{2}}^{-} A_{\mathbf{k}_{3}}^{-} A_{\mathbf{k}_{4}}^{-}\right)\right\} .
\end{aligned}
$$

For the trispectrum for the nonplanar high-momentum modes, we obtain

$$
\begin{aligned}
& \operatorname{Im}\left\{\left(A_{\mathbf{k}_{1}}^{+}-A_{\mathbf{k}_{1}}^{-}\right)^{*}\left(A_{\mathbf{k}_{2}}^{+}-A_{\mathbf{k}_{2}}^{-}\right)^{*}\left(A_{\mathbf{k}_{3}}^{+}-A_{\mathbf{k}_{3}}^{-}\right)^{*}\left(A_{\mathbf{k}_{4}}^{+}-A_{\mathbf{k}_{4}}^{-}\right)^{*}\left(A_{\mathbf{k}_{1}}^{-} A_{\mathbf{k}_{2}}^{+} A_{\mathbf{k}_{3}}^{+} A_{\mathbf{k}_{4}}^{+}+A_{\mathbf{k}_{1}}^{+} A_{\mathbf{k}_{2}}^{-} A_{\mathbf{k}_{3}}^{-} A_{\mathbf{k}_{4}}^{-}\right)\right\} \\
& \simeq \frac{Q\left(r_{\perp, 1}\right)}{2} \frac{H_{0}^{2}}{k_{1}^{2}} .
\end{aligned}
$$

Hence

$$
T_{c}\left(\mathbf{k}_{1}, \mathbf{k}_{2}, \mathbf{k}_{3}, \mathbf{k}_{4}\right) \rightarrow-\frac{3 H_{0}^{10} \eta_{0}^{5}}{10\left(k_{1}^{3} k_{2} k_{3} k_{4}\right)} Q\left(r_{\perp, 1}\right) .
$$

The ratio of Eq. (61) to the case of the de Sitter inflation, Eq. (A2), is given by

$$
k_{1}^{3} H_{0}^{2} \eta_{0}^{5} Q\left(r_{\perp, 1}\right)=\frac{M^{5}}{H_{0}^{5}} \frac{k_{1}^{3} H_{0}^{2}}{k^{5}} Q\left(r_{\perp, 1}\right),
$$

which is of order $\frac{M^{5}}{H_{0}^{5}} \epsilon_{*}^{4}$. Thus the direction dependent trispectrum can be as large as that in the de Sitter inflation for $\frac{M}{H_{0}}>\epsilon_{*}^{-\frac{4}{5}}$. With the condition for the modes Eq. (35), the energy scale for the interaction is bounded as $\epsilon_{*}^{-\frac{4}{5}}<\frac{M}{H_{0}}<\epsilon_{*}^{-1}$.

On the other hand, for the planar modes, the trispectrum in the same limit is given by

$$
T_{c}\left(\mathbf{k}_{1}, \mathbf{k}_{2}, \mathbf{k}_{3}, \mathbf{k}_{4}\right) \rightarrow \frac{3 H_{0}^{8} \eta_{0}^{5}}{5\left(k_{1} k_{2} k_{3} k_{4}\right)} \frac{e^{-\pi q_{1,1}} \sin \left(2 \psi_{1}\right)}{\left(1-e^{-2 \pi q_{1,1}}\right)\left(1-e^{-2 \pi q_{1,2}}\right)\left(1-e^{-2 \pi q_{1,3}}\right)\left(1-e^{-2 \pi q_{1,4}}\right)} .
$$

The ratio to the case of the de Sitter inflation is given by

$$
\frac{M^{5}}{H_{0}^{5}} \frac{k_{1}^{5}}{k^{5}} \frac{e^{-\pi q_{1,1}} \sin \left(2 \psi_{1}\right)}{\left(1-e^{-2 \pi q_{1,1}}\right)\left(1-e^{-2 \pi q_{1,2}}\right)\left(1-e^{-2 \pi q_{1,3}}\right)\left(1-e^{-2 \pi q_{1,4}}\right)},
$$

which is greater than unity since $\frac{M}{H_{0}} \gg 1$. This bound is consistent with Eq. (35) for $1<\frac{M}{H_{0}}<\epsilon_{*}^{-1}$.

Similar results can be obtained for the cases of $k_{2} \rightarrow k_{1}+k_{3}+k_{4}, k_{3} \rightarrow k_{1}+k_{2}+k_{4}$, and $k_{4} \rightarrow k_{1}+k_{2}+k_{3}$. 


\section{Case II}

In the limit $k_{1}+k_{2} \rightarrow k_{3}+k_{4}$, the leading order contribution to the trispectrum is given by

$$
\begin{aligned}
T_{c}\left(\mathbf{k}_{1}, \mathbf{k}_{2}, \mathbf{k}_{3}, \mathbf{k}_{4}\right) & \rightarrow \frac{3 H_{0}^{8} \eta_{0}^{5}}{5\left(k_{1} k_{2} k_{3} k_{4}\right)} \operatorname{Im}\left\{\left(A_{\mathbf{k}_{1}}^{+}-A_{\mathbf{k}_{1}}^{-}\right)^{*}\left(A_{\mathbf{k}_{2}}^{+}-A_{\mathbf{k}_{2}}^{-}\right)^{*}\left(A_{\mathbf{k}_{3}}^{+}-A_{\mathbf{k}_{3}}^{-}\right)^{*}\left(A_{\mathbf{k}_{4}}^{+}-A_{\mathbf{k}_{4}}^{-}\right)^{*}\right. \\
& \left.\times\left(A_{\mathbf{k}_{1}}^{-} A_{\mathbf{k}_{2}}^{-} A_{\mathbf{k}_{3}}^{+} A_{\mathbf{k}_{4}}^{+}+A_{\mathbf{k}_{1}}^{+} A_{\mathbf{k}_{2}}^{+} A_{\mathbf{k}_{3}}^{-} A_{\mathbf{k}_{4}}^{-}\right)\right\} .
\end{aligned}
$$

We see that this type of trispectrum can be much more important than in the previous case. For the trispectrum for the nonplanar high-momentum modes, we obtain

$$
\begin{aligned}
& \operatorname{Im}\left\{\left(A_{\mathbf{k}_{1}}^{+}-A_{\mathbf{k}_{1}}^{-}\right)^{*}\left(A_{\mathbf{k}_{2}}^{+}-A_{\mathbf{k}_{2}}^{-}\right)^{*}\left(A_{\mathbf{k}_{3}}^{+}-A_{\mathbf{k}_{3}}^{-}\right)^{*}\left(A_{\mathbf{k}_{4}}^{+}-A_{\mathbf{k}_{4}}^{-}\right)^{*}\right. \\
\times & \left.\left(A_{\mathbf{k}_{1}}^{-} A_{\mathbf{k}_{2}}^{-} A_{\mathbf{k}_{3}}^{+} A_{\mathbf{k}_{4}}^{+}+A_{\mathbf{k}_{1}}^{+} A_{\mathbf{k}_{2}}^{+} A_{\mathbf{k}_{3}}^{-} A_{\mathbf{k}_{4}}^{-}\right)\right\} \\
\simeq & \frac{Q\left(r_{\perp, 1}\right) Q\left(r_{\perp, 2}\right)}{4} \frac{H_{0}^{7 / 2}}{k_{1}^{3 / 2} k_{2}^{3 / 2}}\left(\frac{1}{k_{1}^{\frac{1}{2}}}+\frac{1}{k_{2}^{\frac{1}{2}}}\right)+\frac{Q\left(r_{\perp, 3}\right) Q\left(r_{\perp, 4}\right)}{4} \frac{H_{0}^{7 / 2}}{k_{3}^{3 / 2} k_{4}^{3 / 2}}\left(\frac{1}{k_{3}^{\frac{1}{2}}}+\frac{1}{k_{4}^{\frac{1}{2}}}\right) .
\end{aligned}
$$

Thus

$$
T_{c}\left(\mathbf{k}_{1}, \mathbf{k}_{2}, \mathbf{k}_{3}, \mathbf{k}_{4}\right) \rightarrow \frac{3 H_{0}^{\frac{23}{2}} \eta_{0}^{5}}{20\left(k_{1} k_{2} k_{3} k_{4}\right)}\left[\frac{Q\left(r_{\perp, 1}\right) Q\left(r_{\perp, 2}\right)}{k_{1}^{\frac{3}{2}} k_{2}^{\frac{3}{2}}}\left(\frac{1}{k_{1}^{\frac{1}{2}}}+\frac{1}{k_{2}^{\frac{1}{2}}}\right)+\frac{Q\left(r_{\perp, 3}\right) Q\left(r_{\perp, 4}\right)}{k_{3}^{\frac{3}{2}} k_{4}^{\frac{3}{2}}}\left(\frac{1}{k_{3}^{\frac{1}{2}}}+\frac{1}{k_{4}^{\frac{1}{2}}}\right)\right]
$$

The ratio of Eq. (67) to the case of the de Sitter inflation Eq. (A2) is given by

$$
\begin{aligned}
& \left(k_{1}+k_{2}\right)^{5} \eta_{0}^{5} H_{0}^{\frac{7}{2}}\left[\frac{Q\left(r_{\perp, 1}\right) Q\left(r_{\perp, 2}\right)}{k_{1}^{\frac{3}{2}} k_{2}^{\frac{3}{2}}}\left(\frac{1}{k_{1}^{\frac{1}{2}}}+\frac{1}{k_{2}^{\frac{1}{2}}}\right)+\frac{Q\left(r_{\perp, 3}\right) Q\left(r_{\perp, 4}\right)}{k_{3}^{\frac{3}{2}} k_{4}^{\frac{3}{2}}}\left(\frac{1}{k_{3}^{\frac{1}{2}}}+\frac{1}{k_{4}^{\frac{1}{2}}}\right)\right] \\
= & \frac{M^{5}}{H_{0}^{5}} \frac{\left(k_{1}+k_{2}\right)^{5} H_{0}^{\frac{7}{2}}}{k^{5}}\left[\frac{Q\left(r_{\perp, 1}\right) Q\left(r_{\perp, 2}\right)}{k_{1}^{\frac{3}{2}} k_{2}^{\frac{3}{2}}}\left(\frac{1}{k_{1}^{\frac{1}{2}}}+\frac{1}{k_{2}^{\frac{1}{2}}}\right)+\frac{Q\left(r_{\perp, 3}\right) Q\left(r_{\perp, 4}\right)}{k_{3}^{\frac{3}{2}}} k_{4}^{\frac{3}{2}}\left(\frac{1}{k_{3}^{\frac{1}{2}}}+\frac{1}{k_{4}^{\frac{1}{2}}}\right)\right],
\end{aligned}
$$

which is of order $\frac{M^{5}}{H_{0}^{5}} \epsilon_{*}^{7}$. Thus the direction dependent trispectrum can be as large as that in the de Sitter inflation for $\frac{M}{H_{0}}>\epsilon_{*}^{-\frac{7}{5}}$. But this is never satisfied because of Eq. (35). In addition, assuming that $k=k_{1}+k_{2}+k_{3}+k_{4}$ is fixed, the ratio of the trispectrum of case II, Eq. (67), to that of case I, Eq. (61), is given by $\left(\frac{H_{0}}{k}\right)^{\frac{3}{2}}$, which is suppressed.

On the other hand, for the planar modes, the trispectrum in the same limit is given by

$$
\begin{aligned}
T_{c}\left(\mathbf{k}_{1}, \mathbf{k}_{2}, \mathbf{k}_{3}, \mathbf{k}_{4}\right) & \rightarrow-\frac{3 H_{0}^{8} \eta_{0}^{5}}{5\left(k_{1} k_{2} k_{3} k_{4}\right)} \\
& \times \frac{e^{-\pi\left(q_{1,1}+q_{1,2}\right)} \sin \left(2 \psi_{1}+2 \psi_{2}\right)+e^{-\pi\left(q_{1,3}+q_{1,4}\right)} \sin \left(2 \psi_{3}+2 \psi_{4}\right)}{\left(1-e^{-2 \pi q_{1,1}}\right)\left(1-e^{-2 \pi q_{1,2}}\right)\left(1-e^{-2 \pi q_{1,3}}\right)\left(1-e^{-2 \pi q_{1,4}}\right)} .
\end{aligned}
$$

The ratio to the case of the de Sitter inflation is given by

$$
\frac{M^{5}}{H_{0}^{5}} \frac{\left(k_{1}+k_{2}\right)^{5}}{k^{5}} \frac{e^{-\pi\left(q_{1,1}+q_{1,2}\right)} \sin \left(2 \psi_{1}+2 \psi_{2}\right)+e^{-\pi\left(q_{1,3}+q_{1,4}\right)} \sin \left(2 \psi_{3}+2 \psi_{4}\right)}{\left(1-e^{-2 \pi q_{1,1}}\right)\left(1-e^{-2 \pi q_{1,2}}\right)\left(1-e^{-2 \pi q_{1,3}}\right)\left(1-e^{-2 \pi q_{1,4}}\right)},
$$

which is greater than unity since $\frac{M}{H_{0}} \gg 1$. This bound is consistent with Eq. (355) for $1<\frac{M}{H_{0}}<\epsilon_{*}^{-1}$. The ratio of Eq. (63) to Eq. (69) is given by

$$
\frac{e^{-\pi q_{1,1}} \sin \left(2 \psi_{1}\right)}{e^{-\pi\left(q_{1,1}+q_{1,2}\right)} \sin \left(2 \psi_{1}+2 \psi_{2}\right)+e^{-\pi\left(q_{1,3}+q_{1,4}\right)} \sin \left(2 \psi_{3}+2 \psi_{4}\right)},
$$

which is of order unity, in contrast to the one for the nonplanar high-momentum modes.

Similar results can be obtained for the case of $k_{1}+k_{4} \rightarrow k_{2}+k_{3}$. 
3. Case III

In the squeezed limit of $k_{1}=k_{2} \gg k_{12}$ and $k_{3}=k_{4} \gg k_{12}$, the leading order contribution to the trispectrum is given by

$$
\begin{aligned}
T_{c}\left(\mathbf{k}_{1}, \mathbf{k}_{2}, \mathbf{k}_{3}, \mathbf{k}_{4}\right) & \rightarrow \frac{3 H_{0}^{8} \eta_{0}^{5}}{5\left(k_{1}^{2} k_{3}^{2}\right)} \operatorname{Im}\left\{\left(A_{\mathbf{k}_{1}}^{+}-A_{\mathbf{k}_{1}}^{-}\right)^{*}\left(A_{\mathbf{k}_{2}}^{+}-A_{\mathbf{k}_{2}}^{-}\right)^{*}\left(A_{\mathbf{k}_{3}}^{+}-A_{\mathbf{k}_{3}}^{-}\right)^{*}\left(A_{\mathbf{k}_{4}}^{+}-A_{\mathbf{k}_{4}}^{-}\right)^{*}\right. \\
& \left.\times\left(A_{\mathbf{k}_{1}}^{+} A_{\mathbf{k}_{2}}^{-}+A_{\mathbf{k}_{1}}^{-} A_{\mathbf{k}_{2}}^{+}\right)\left(A_{\mathbf{k}_{3}}^{+} A_{\mathbf{k}_{4}}^{-}+A_{\mathbf{k}_{3}}^{-} A_{\mathbf{k}_{4}}^{+}\right)^{*}\right\} \\
& \simeq \frac{3 H_{0}^{\frac{23}{2}} \eta_{0}^{5}}{20\left(k_{1}^{\frac{7}{2}} k_{3}^{\frac{7}{2}}\right.}\left(\frac{1}{k_{1}^{\frac{1}{2}}}+\frac{1}{k_{3}^{\frac{1}{2}}}\right)\left(Q\left(r_{\perp, 1}\right)+Q\left(r_{\perp, 2}\right)\right)\left(Q\left(r_{\perp, 3}\right)+Q\left(r_{\perp, 4}\right)\right) .
\end{aligned}
$$

The ratio of Eq. (172) to that in the case of the de Sitter inflation, Eq. (A2), is given by

$$
\begin{aligned}
& \frac{H_{0}^{\frac{7}{2}} \eta_{0}^{5}\left(k_{1}+k_{2}\right)^{5}}{k_{1}^{\frac{3}{2}} k_{3}^{\frac{3}{2}}}\left(\frac{1}{k_{1}^{\frac{1}{2}}}+\frac{1}{k_{3}^{\frac{1}{2}}}\right)\left(Q\left(r_{\perp, 1}\right)+Q\left(r_{\perp, 2}\right)\right)\left(Q\left(r_{\perp, 3}\right)+Q\left(r_{\perp, 4}\right)\right) \\
= & \frac{M^{5}}{H_{0}^{5}} \frac{H_{0}^{\frac{7}{2}}\left(k_{1}+k_{2}\right)^{5}}{k_{1}^{\frac{3}{2}} k_{3}^{\frac{3}{2}}} k^{5}\left(\frac{1}{k_{1}^{\frac{1}{2}}}+\frac{1}{k_{3}^{\frac{1}{2}}}\right)\left(Q\left(r_{\perp, 1}\right)+Q\left(r_{\perp, 2}\right)\right)\left(Q\left(r_{\perp, 3}\right)+Q\left(r_{\perp, 4}\right)\right),
\end{aligned}
$$

which is of order $\frac{M^{5}}{H_{0}^{5}} \epsilon_{*}^{7}$. Thus the direction dependent trispectrum can be as large as that in the de Sitter inflation for $\frac{M}{H_{0}}>\epsilon_{*}^{-\frac{7}{5}}$. But this is never satisfied because of Eq. (35). In addition, assuming that $k=k_{1}+k_{2}+k_{3}+k_{4}$ is fixed, the ratio of the trispectrum of case II, Eq. (67), to that of case III, Eq. (72), is of order $O(1)$, and the ratio to case I, 61], is suppressed by $\epsilon_{*}^{3}$.

On the other hand, for the planar modes,

$$
\begin{aligned}
T_{c}\left(\mathbf{k}_{1}, \mathbf{k}_{2}, \mathbf{k}_{3}, \mathbf{k}_{4}\right) & \rightarrow-\frac{3 H_{0}^{8} \eta_{0}^{5}}{5\left(k_{1}^{2} k_{3}^{2}\right)} \frac{1}{\left(1-e^{-\pi q_{1,1}}\right)\left(1-e^{-\pi q_{1,2}}\right)\left(1-e^{-\pi q_{1,3}}\right)\left(1-e^{-\pi q_{1,4}}\right)} \\
& \times\left[e^{-\pi\left(q_{1,1}+q_{1,3}\right)} \sin \left(2 \psi_{1}+2 \psi_{3}\right)+e^{-\pi\left(q_{1,1}+q_{1,4}\right)} \sin \left(2 \psi_{1}+2 \psi_{4}\right)\right. \\
& \left.+e^{-\pi\left(q_{1,2}+q_{1,3}\right)} \sin \left(2 \psi_{2}+2 \psi_{3}\right)+e^{-\pi\left(q_{1,2}+q_{1,4}\right)} \sin \left(2 \psi_{2}+2 \psi_{4}\right)\right] .
\end{aligned}
$$

The ratio to the case of the de Sitter inflation is given by

$$
\begin{aligned}
& \frac{M^{5}}{H_{0}^{5}} \frac{\left(k_{1}+k_{3}\right)^{5}}{k^{5}} \frac{1}{\left(1-e^{-\pi q_{1,1}}\right)\left(1-e^{-\pi q_{1,2}}\right)\left(1-e^{-\pi q_{1,3}}\right)\left(1-e^{-\pi q_{1,4}}\right)} \\
\times & {\left[e^{-\pi\left(q_{1,1}+q_{1,3}\right)} \sin \left(2 \psi_{1}+2 \psi_{3}\right)+e^{-\pi\left(q_{1,1}+q_{1,4}\right)} \sin \left(2 \psi_{1}+2 \psi_{4}\right)\right.} \\
+ & \left.e^{-\pi\left(q_{1,2}+q_{1,3}\right)} \sin \left(2 \psi_{2}+2 \psi_{3}\right)+e^{-\pi\left(q_{1,2}+q_{1,4}\right)} \sin \left(2 \psi_{2}+2 \psi_{4}\right)\right],
\end{aligned}
$$

which is greater than unity since $\frac{M}{H_{0}} \gg 1$. This bound is consistent with Eq. (35) for $1<\frac{M}{H_{0}}<\epsilon_{*}^{-1}$. The ratio of Eq. (69) to Eq. (74) is given by

$$
\begin{aligned}
& {\left[e^{-\pi\left(q_{1,1}+q_{1,2}\right)} \sin \left(2 \psi_{1}+2 \psi_{2}\right)+e^{-\pi\left(q_{1,3}+q_{1,4}\right)} \sin \left(2 \psi_{3}+2 \psi_{4}\right)\right] } \\
\times & {\left[e^{-\pi\left(q_{1,1}+q_{1,3}\right)} \sin \left(2 \psi_{1}+2 \psi_{3}\right)+e^{-\pi\left(q_{1,1}+q_{1,4}\right)} \sin \left(2 \psi_{1}+2 \psi_{4}\right)\right.} \\
+ & \left.e^{-\pi\left(q_{1,2}+q_{1,3}\right)} \sin \left(2 \psi_{2}+2 \psi_{3}\right)+e^{-\pi\left(q_{1,2}+q_{1,4}\right)} \sin \left(2 \psi_{2}+2 \psi_{4}\right)\right]^{-1}
\end{aligned}
$$

which is of order unity, in contrast to the one for the nonplanar high-momentum modes.

Similar results can be obtained for the case of $k_{1}=k_{4} \gg k_{14}$ and $k_{2}=k_{3} \gg k_{14}$. 


\section{Features of the trispectra from the contact interaction}

We summarize the features of the trispectra from the contact interaction diagram and compare them with those in the de Sitter inflation. In our background the trispectrum in the limiting case I (for example, for $k_{1} \rightarrow k_{2}+k_{3}+k_{4}$ ) is greater than those in the limiting case II ( for example, for $k_{1}+k_{2} \rightarrow k_{3}+k_{4}$ ) and case III (for example, for $k_{1}=k_{2} \gg k_{12}$ and $k_{3}=k_{4} \gg k_{12}$ ), by a factor of $\left(\frac{k}{H_{0}}\right)^{\frac{3}{2}}$, where $k \gg H_{0}$ is the typical size of the momentum vectors.

We then summarize the direction dependence of the trispectra. Though we focus on the nonplanar high-momentum modes, the essence is the same for the planar modes. In the limiting case I, for example, for $k_{1} \rightarrow k_{2}+k_{3}+k_{4}$, the trispectrum is proportional to $Q\left(r_{\perp, 1}\right)$ and hence depends on the direction of the momentum vector $\mathbf{k}_{1}$. In the limiting case II, for example, for $k_{1}+k_{2} \rightarrow k_{3}+k_{4}$, it is proportional to a combination of $Q\left(r_{\perp, 1}\right) Q\left(r_{\perp, 2}\right)$ and $Q\left(r_{\perp, 3}\right) Q\left(r_{\perp, 4}\right)$, which depends on the directions of all the momenta. In the limiting case III, for example, for $k_{1}=k_{2} \gg k_{12}$ and $k_{3}=k_{4} \gg k_{12}$, the trispectrum is proportional to $\left(Q\left(r_{\perp, 1}\right)+Q\left(r_{\perp, 2}\right)\right)\left(Q\left(r_{\perp, 3}\right)+Q\left(r_{\perp, 4}\right)\right)$, which depends on the directions of all the external momenta. Thus the trispectra are direction dependent, and the dependence is significantly different in various limiting cases.

We finally compare the amplitudes of the trispectra from a contact interaction diagram with those in the de Sitter inflation. The amplitude of the trispectra for the planar modes in all the limiting cases I, II and III can be greater than those in the de Sitter inflation. For the nonplanar high-momentum modes, the amplitude of the trispectrum in the limiting case I can be as large as that in the de Sitter inflation, while those in the other two limiting cases cannot be as large as those in the de Sitter inflation. Thus, combined with the direction dependence, the trispectrum in the limiting case I for the nonplanar high-momentum modes, as well as those in all the limiting cases for the planar modes, would be particularly interesting to distinguish models.

\section{B. Contribution from the scalar exchange interaction}

The other leading order contribution to the trispectrum is given by the scalar exchange interaction. The trispectrum is given by

$$
\begin{aligned}
& \left\langle\phi\left(t, \mathbf{k}_{1}\right) \phi\left(t, \mathbf{k}_{2}\right) \phi\left(t, \mathbf{k}_{3}\right) \phi\left(t, \mathbf{k}_{4}\right)\right\rangle \\
= & -\int_{t_{0}}^{t} d t_{2} \int_{t_{0}}^{t_{2}} d t_{1}\left\langle\left[H_{I}^{(3)}\left(t_{1}\right),\left[H_{I}^{(3)}\left(t_{2}\right), \phi^{I}\left(t, \mathbf{k}_{1}\right) \phi^{I}\left(t, \mathbf{k}_{2}\right) \phi^{I}\left(t, \mathbf{k}_{3}\right) \phi^{I}\left(t, \mathbf{k}_{4}\right)\right]\right]\right\rangle,
\end{aligned}
$$

where the interaction Hamiltonian is given by Eq. (34). The trispectrum in the $t \rightarrow 0$ limit takes the following form

$$
\left\langle\phi\left(0, \mathbf{k}_{1}\right) \phi\left(0, \mathbf{k}_{2}\right) \phi\left(0, \mathbf{k}_{3}\right) \phi\left(0, \mathbf{k}_{4}\right)\right\rangle=(2 \pi)^{-3} \frac{q_{3}^{2}}{M^{4}} T_{s}\left(\mathbf{k}_{1}, \mathbf{k}_{2}, \mathbf{k}_{3}, \mathbf{k}_{4}\right) \delta\left(\mathbf{k}_{1}+\mathbf{k}_{2}+\mathbf{k}_{3}+\mathbf{k}_{4}\right) .
$$


After computations, we obtain

$$
\begin{aligned}
T_{s}\left(\mathbf{k}_{1}, \mathbf{k}_{2}, \mathbf{k}_{3}, \mathbf{k}_{4}\right) & =\frac{9 H_{0}^{8}}{4\left(k_{1} k_{2} k_{3} k_{4}\right)} \\
& \times \operatorname{Re}\left\{k_{12}\left(A_{\mathbf{k}_{1}}^{+}-A_{\mathbf{k}_{1}}^{-}\right)\left(A_{\mathbf{k}_{2}}^{+}-A_{\mathbf{k}_{2}}^{-}\right)\left(A_{\mathbf{k}_{3}}^{+}-A_{\mathbf{k}_{3}}^{-}\right)^{*}\left(A_{\mathbf{k}_{4}}^{+}-A_{\mathbf{k}_{4}}^{-}\right)^{*}\right. \\
& \times\left[-A_{\mathbf{k}_{3}}^{+} A_{\mathbf{k}_{4}}^{+} A_{\mathbf{k}_{12}}^{+} \mathcal{I}\left(k_{3}, k_{4}, k_{12}\right)+A_{\mathbf{k}_{3}}^{-} A_{\mathbf{k}_{4}}^{-} A_{\mathbf{k}_{12}}^{-} \mathcal{I}\left(-k_{3},-k_{4},-k_{12}\right)\right. \\
& +\left(A_{\mathbf{k}_{3}}^{+} A_{\mathbf{k}_{4}}^{+} A_{\mathbf{k}_{12}}^{-} \mathcal{I}\left(k_{3}, k_{4},-k_{12}\right)+2 \text { perms }\right) \\
& \left.-\left(A_{\mathbf{k}_{3}}^{+} A_{\mathbf{k}_{4}}^{-} A_{\mathbf{k}_{12}}^{-} \mathcal{I}\left(k_{3},-k_{4},-k_{12}\right)+2 \text { perms }\right)\right] \\
& \times\left[-\left(A_{\mathbf{k}_{1}}^{+} A_{\mathbf{k}_{2}}^{+} A_{\mathbf{k}_{12}}^{+}\right)^{*} \mathcal{I}\left(-k_{1},-k_{2},-k_{12}\right)+\left(A_{\mathbf{k}_{1}}^{-} A_{\mathbf{k}_{2}}^{-} A_{\mathbf{k}_{12}}^{-}\right)^{*} \mathcal{I}\left(k_{1}, k_{2}, k_{12}\right)\right. \\
& +\left(\left(A_{\mathbf{k}_{1}}^{+} A_{\mathbf{k}_{2}}^{+} A_{\mathbf{k}_{12}}^{-}\right)^{*} \mathcal{I}\left(-k_{1},-k_{2}, k_{12}\right)+2 \text { perms }\right) \\
& \left.-\left(\left(A_{\mathbf{k}_{1}}^{+} A_{\mathbf{k}_{2}}^{-} A_{\mathbf{k}_{12}}^{-}\right)^{*} \mathcal{I}\left(-k_{1}, k_{2}, k_{12}\right)+2 \text { perms }\right)\right] \\
& +k_{14}\left(A_{\mathbf{k}_{2}}^{+}-A_{\mathbf{k}_{2}}^{-}\right)\left(A_{\mathbf{k}_{3}}^{+}-A_{\mathbf{k}_{3}}^{-}\right)\left(A_{\mathbf{k}_{4}}^{+}-A_{\mathbf{k}_{4}}^{-}\right)^{*}\left(A_{\mathbf{k}_{1}}^{+}-A_{\mathbf{k}_{1}}^{-}\right)^{*} \\
& \times\left[-A_{\mathbf{k}_{4}}^{+} A_{\mathbf{k}_{1}}^{+} A_{\mathbf{k}_{14}}^{+} \mathcal{I}\left(k_{4}, k_{1}, k_{14}\right)+A_{\mathbf{k}_{4}}^{-} A_{\mathbf{k}_{1}}^{-} A_{\mathbf{k}_{14}}^{-} \mathcal{I}\left(-k_{4},-k_{1},-k_{14}\right)\right. \\
& +\left(A_{\mathbf{k}_{4}}^{+} A_{\mathbf{k}_{1}}^{+} A_{\mathbf{k}_{14}}^{-} \mathcal{I}\left(k_{3}, k_{4},-k_{14}\right)+2 \text { perms }\right) \\
& \left.-\left(A_{\mathbf{k}_{4}}^{+} A_{\mathbf{k}_{1}}^{-} A_{\mathbf{k}_{14}}^{-} \mathcal{I}\left(k_{4},-k_{1},-k_{14}\right)+2 \text { perms }\right)\right] \\
& \times\left[-\left(A_{\mathbf{k}_{2}}^{+} A_{\mathbf{k}_{3}}^{+} A_{\mathbf{k}_{14}}^{+}\right)^{*} \mathcal{I}\left(-k_{2},-k_{3},-k_{14}\right)+\left(A_{\mathbf{k}_{2}}^{-} A_{\mathbf{k}_{3}}^{-} A_{\mathbf{k}_{14}}^{-}\right)^{*} \mathcal{I}\left(k_{2}, k_{3}, k_{14}\right)\right. \\
& +\left(\left(A_{\mathbf{k}_{2}}^{+} A_{\mathbf{k}_{3}}^{+} A_{\mathbf{k}_{14}}^{-}\right)^{*} \mathcal{I}\left(-k_{2},-k_{3}, k_{14}\right)+2 \text { perms }\right) \\
& \left.\left.-\left(\left(A_{\mathbf{k}_{2}}^{+} A_{\mathbf{k}_{3}}^{-} A_{\mathbf{k}_{14}}^{-}\right)^{*} \mathcal{I}\left(-k_{2}, k_{3}, k_{14}\right)+2 \text { perms }\right)\right]\right\} \\
& s
\end{aligned}
$$

Thus the trispectrum from the scalar exchange interaction depends on $k_{12}=\left|\mathbf{k}_{1}+\mathbf{k}_{2}\right|$ and $k_{14}=\left|\mathbf{k}_{1}+\mathbf{k}_{4}\right|$ as well as on $k_{i}(i=1,2,3,4)$ because of the internal line in the diagram. Since $\left|\mathcal{I}\left(p_{1}, p_{2}, p_{3}\right)\right|$ is increasing in the limit of $p_{1}+p_{2}+p_{3} \rightarrow 0$, the bispectrum has the maximum amplitude.

We focus on limits $k_{1}+k_{2} \rightarrow k_{12}$ and $k_{3}+k_{4} \rightarrow k_{12}$, and $k_{1}+k_{4} \rightarrow k_{14}$ and $k_{2}+k_{3} \rightarrow k_{14}$ (case I). We also investigate the limiting cases of $k_{1}=k_{2} \gg k_{12}$ and $k_{3}=k_{4} \gg k_{12}$, and $k_{1}=k_{4} \gg k_{14}$ and $k_{2}=k_{3} \gg k_{14}$ (case II).

\section{Case $I$}

In the limit of $k_{1}+k_{2} \rightarrow k_{12}$ and $k_{3}+k_{4} \rightarrow k_{12}$, the leading order contribution to the trispectrum is given by

$$
\begin{aligned}
T_{s}\left(\mathbf{k}_{1}, \mathbf{k}_{2}, \mathbf{k}_{3}, \mathbf{k}_{4}\right) & \rightarrow \frac{H_{0}^{8} k_{12} \eta_{0}^{6}}{4\left(k_{1} k_{2} k_{3} k_{4}\right)} \\
& \times \operatorname{Re}\left\{\left(A_{\mathbf{k}_{1}}^{+}-A_{\mathbf{k}_{1}}^{-}\right)\left(A_{\mathbf{k}_{2}}^{+}-A_{\mathbf{k}_{2}}^{-}\right)\left(A_{\mathbf{k}_{3}}^{+}-A_{\mathbf{k}_{3}}^{-}\right)^{*}\left(A_{\mathbf{k}_{4}}^{+}-A_{\mathbf{k}_{4}}^{-}\right)^{*}\right. \\
& \left.\times\left[A_{\mathbf{k}_{3}}^{+} A_{\mathbf{k}_{4}}^{+} A_{\mathbf{k}_{12}}^{-}-A_{\mathbf{k}_{3}}^{-} A_{\mathbf{k}_{4}}^{-} A_{\mathbf{k}_{12}}^{+}\right]\left[\left(A_{\mathbf{k}_{1}}^{+} A_{\mathbf{k}_{2}}^{+} A_{\mathbf{k}_{12}}^{-}\right)^{*}-\left(A_{\mathbf{k}_{1}}^{-} A_{\mathbf{k}_{2}}^{-} A_{\mathbf{k}_{12}}^{+}\right)^{*}\right]\right\} .
\end{aligned}
$$

For the trispectrum of the nonplanar high-momentum modes, we obtain

$$
\begin{aligned}
& \operatorname{Re}\left\{\left(A_{\mathbf{k}_{1}}^{+}-A_{\mathbf{k}_{1}}^{-}\right)\left(A_{\mathbf{k}_{2}}^{+}-A_{\mathbf{k}_{2}}^{-}\right)\left(A_{\mathbf{k}_{3}}^{+}-A_{\mathbf{k}_{3}}^{-}\right)^{*}\left(A_{\mathbf{k}_{4}}^{+}-A_{\mathbf{k}_{4}}^{-}\right)^{*}\right. \\
\times & {\left.\left[A_{\mathbf{k}_{3}}^{+} A_{\mathbf{k}_{4}}^{+} A_{\mathbf{k}_{12}}^{-}-A_{\mathbf{k}_{3}}^{-} A_{\mathbf{k}_{4}}^{-} A_{\mathbf{k}_{12}}^{+}\right]\left[\left(A_{\mathbf{k}_{1}}^{+} A_{\mathbf{k}_{2}}^{+} A_{\mathbf{k}_{12}}^{-}\right)^{*}-\left(A_{\mathbf{k}_{1}}^{-} A_{\mathbf{k}_{2}}^{-} A_{\mathbf{k}_{12}}^{+}\right)^{*}\right]\right\} \simeq \frac{Q\left(r_{\perp, 12}\right)^{2} H_{0}^{3}}{4 k_{12}^{3}} . }
\end{aligned}
$$

Thus

$$
T_{s}\left(\mathbf{k}_{1}, \mathbf{k}_{2}, \mathbf{k}_{3}, \mathbf{k}_{4}\right) \rightarrow \frac{H_{0}^{11} \eta_{0}^{6} Q\left(r_{\perp, 12}\right)^{2}}{16\left(k_{1} k_{2} k_{3} k_{4}\right) k_{12}^{2}}
$$


The ratio to the case of the de Sitter inflation, Eq. (A3), is given by

$$
k_{12}^{3} H_{0}^{3} \eta_{0}^{6} Q\left(r_{\perp, 12}\right)^{2}=\frac{M^{6}}{H_{0}^{6}} \frac{k_{12}^{3} H_{0}^{3}}{k^{6}} Q\left(r_{\perp, 12}\right)^{2},
$$

which is of order $\frac{M^{6}}{H_{0}^{6}} \epsilon_{*}^{6}$. Thus the direction dependent trispectrum can be as large as that in the de Sitter inflation for $\frac{M}{H_{0}}>\epsilon_{*}^{-1}$. This is marginally consistent with Eq. (35).

On the other hand, the trispectrum in the same limit is given by

$$
T_{s}\left(\mathbf{k}_{1}, \mathbf{k}_{2}, \mathbf{k}_{3}, \mathbf{k}_{4}\right) \rightarrow \frac{H_{0}^{8} k_{12} \eta_{0}^{6}}{4\left(k_{1} k_{2} k_{3} k_{4}\right)} \frac{e^{-2 \pi q_{1,12}}}{\left(1-e^{-2 \pi q_{1,1}}\right)\left(1-e^{-2 \pi q_{1,2}}\right)\left(1-e^{-2 \pi q_{1,3}}\right)\left(1-e^{-2 \pi q_{1,4}}\right)\left(1-e^{-2 \pi q_{1,12}}\right)} .
$$

The ratio to the case of the de Sitter inflation is given by

$$
\frac{M^{6}}{H_{0}^{6}} \frac{k_{12}^{6}}{k^{6}} \frac{e^{-2 \pi q_{1,1}}}{\left(1-e^{-\pi q_{1,1}}\right)\left(1-e^{-\pi q_{1,2}}\right)\left(1-e^{-\pi q_{1,3}}\right)\left(1-e^{-\pi q_{1,4}}\right)},
$$

which is greater than unity since $\frac{M}{H_{0}} \gg 1$. This bound is consistent with Eq. (35) for $1<\frac{M}{H_{0}}<\epsilon_{*}^{-1}$.

Similar results can be obtained for $k_{1}+k_{4} \rightarrow k_{14}$ and $k_{2}+k_{3} \rightarrow k_{14}$.

\section{Case II}

In the limit of $k_{1}=k_{2} \gg k_{12}$ and $k_{3}=k_{4} \gg k_{12}$, the leading order contribution from the nonplanar high momentum modes is given by

$$
T_{s}\left(\mathbf{k}_{1}, \mathbf{k}_{2}, \mathbf{k}_{3}, \mathbf{k}_{4}\right) \rightarrow \frac{H_{0}^{11} \eta_{0}^{6} k_{12}}{16\left(k_{1}^{\frac{7}{2}} k_{3}^{\frac{7}{2}}\right)}\left(Q\left(r_{\perp, 1}\right)+Q\left(r_{\perp, 2}\right)\right)\left(Q\left(r_{\perp, 3}\right)+Q\left(r_{\perp, 4}\right)\right) .
$$

which is of the same order as Eq. (82). The ratio to the case of the de Sitter inflation, Eq. (A3), is given by

$$
\begin{aligned}
& \left(k_{1} k_{3}\right)^{\frac{3}{2}} H_{0}^{3} \eta_{0}^{6}\left(Q\left(r_{\perp, 1}\right)+Q\left(r_{\perp, 2}\right)\right)\left(Q\left(r_{\perp, 3}\right)+Q\left(r_{\perp, 4}\right)\right) \\
= & \frac{M^{6}}{H_{0}^{6}} \frac{\left(k_{1} k_{3}\right)^{\frac{3}{2}} H_{0}^{3}}{k^{6}}\left(Q\left(r_{\perp, 1}\right)+Q\left(r_{\perp, 2}\right)\right)\left(Q\left(r_{\perp, 3}\right)+Q\left(r_{\perp, 4}\right)\right),
\end{aligned}
$$

which is of order $\frac{M^{6}}{H_{0}^{6}} \epsilon_{*}^{6}$. Thus the direction dependent trispectrum can be as large as that in the de Sitter inflation for $\frac{M}{H_{0}}>\epsilon_{*}^{-1}$. This is marginally consistent with Eq. (35). In addition, the trispectrum in case II, Eq. (86), is suppressed to that in case I, Eq. (82), by the ratio of $\frac{k_{12}}{k} \ll 1$. Thus in contrast to the case of the bispectrum, the trispectrum in case I becomes greater than that in case II.

On the other hand, for the planar modes,

$$
\begin{aligned}
T_{s}\left(\mathbf{k}_{1}, \mathbf{k}_{2}, \mathbf{k}_{3}, \mathbf{k}_{4}\right) & \rightarrow \frac{H_{0}^{8} \eta_{0}^{6}}{4\left(k_{1}^{2} k_{3}^{2}\right)} \frac{k_{12}}{\left(1-e^{-\pi q_{1,1}}\right)\left(1-e^{-\pi q_{1,2}}\right)\left(1-e^{-\pi q_{1,3}}\right)\left(1-e^{-\pi q_{1,4}}\right)\left(1-e^{-\pi q_{1,12}}\right)} \\
& \times\left\{e^{-\pi\left(q_{1,1}+q_{1,3}\right)} \cos \left(2 \psi_{1}-2 \psi_{3}\right)+e^{-\pi\left(q_{1,1}+q_{1,4}\right)} \cos \left(2 \psi_{1}-2 \psi_{4}\right)\right. \\
& \left.+e^{-\pi\left(q_{1,2}+q_{1,3}\right)} \cos \left(2 \psi_{2}-2 \psi_{3}\right)+e^{-\pi\left(q_{1,2}+q_{1,4}\right)} \cos \left(2 \psi_{2}-2 \psi_{4}\right)\right\} .
\end{aligned}
$$

The ratio to the case of the de Sitter inflation is given by

$$
\begin{aligned}
& \frac{M^{6}}{H_{0}^{6}} \frac{k_{1}^{3} k_{3}^{3}}{k^{6}} \frac{1}{\left(1-e^{-\pi q_{1,1}}\right)\left(1-e^{-\pi q_{1,2}}\right)\left(1-e^{-\pi q_{1,3}}\right)\left(1-e^{-\pi q_{1,4}}\right)\left(1-e^{-\pi q_{1,12}}\right)} \\
\times & \left\{e^{-\pi\left(q_{1,1}+q_{1,3}\right)} \cos \left(2 \psi_{1}-2 \psi_{3}\right)+e^{-\pi\left(q_{1,1}+q_{1,4}\right)} \cos \left(2 \psi_{1}-2 \psi_{4}\right)\right. \\
+ & \left.e^{-\pi\left(q_{1,2}+q_{1,3}\right)} \cos \left(2 \psi_{2}-2 \psi_{3}\right)+e^{-\pi\left(q_{1,2}+q_{1,4}\right)} \cos \left(2 \psi_{2}-2 \psi_{4}\right)\right\}
\end{aligned}
$$


which is greater than unity since $\frac{M}{H_{0}} \gg 1$. This bound is consistent with Eq. (35) for $1<\frac{M}{H_{0}}<\epsilon_{*}^{-1}$. The ratio of Eq. (84) to Eq. (88) is given by

$$
\begin{aligned}
& \frac{k e^{-2 \pi q_{1,12}}}{k_{12}}\left[e^{-\pi\left(q_{1,1}+q_{1,3}\right)} \cos \left(2 \psi_{1}-2 \psi_{3}\right)+e^{-\pi\left(q_{1,1}+q_{1,4}\right)} \cos \left(2 \psi_{1}-2 \psi_{4}\right)\right. \\
+ & \left.e^{-\pi\left(q_{1,2}+q_{1,3}\right)} \cos \left(2 \psi_{2}-2 \psi_{3}\right)+e^{-\pi\left(q_{1,2}+q_{1,4}\right)} \cos \left(2 \psi_{2}-2 \psi_{4}\right)\right]^{-1},
\end{aligned}
$$

which can be larger than unity for $k_{12} \ll k$ as for the nonplanar high momentum modes. The result is in contrast to the case of the bispectrum from the contact diagram.

Similar results can be obtained for the case of $k_{1}=k_{4} \gg k_{14}$ and $k_{2}=k_{3} \gg k_{14}$.

\section{Features of the trispectra from the scalar exchange interaction}

We summarize the features of the trispectra from the scalar exhange interaction diagram and compare them with those in the de Sitter inflation. In our background the amplitude of the trispectrum in the limit of case I (for example, for $k_{1}+k_{2} \rightarrow k_{12}$ and $k_{3}+k_{4} \rightarrow k_{12}$ ) is greater than those in the limits of case II (for example, for $k_{1}=k_{2} \gg k_{12}$ and $k_{3}=k_{4} \gg k_{12}$ ), by a factor of $\frac{k}{k_{12}}$, where $k \gg H_{0}$ is the typical size of the momentum vectors.

We then summarize the direction dependence of the trispectra. Though we focus on the nonplanar high-momentum modes, the essence is the same for the planar modes. In the limiting case I, for example, for $k_{1}+k_{2} \rightarrow k_{12}$ and $\left.k_{3}+k_{4} \rightarrow k_{12}\right)$, the trispectrum is proportional to $Q\left(r_{\perp, 12}\right)^{2}$ and hence depends on the direction of the internal momentum vector $\mathbf{k}_{12}$. In the limiting case II, for example, for $k_{1}=k_{2} \gg k_{12}$ and $k_{3}=k_{4} \gg k_{12}$, it is proportional to $\left(Q\left(r_{\perp, 1}\right)+Q\left(r_{\perp, 2}\right)\right)\left(Q\left(r_{\perp, 3}\right)+Q\left(r_{\perp, 4}\right)\right)$, which depends on the directions of all the momenta. Thus the trispectra are direction dependent, and the dependence is significantly different in various limiting cases.

We finally compare the amplitudes of the trispectra from the scalar exchange interaction diagram with those in the de Sitter inflation. The amplitudes of the trispectra from the planar modes in all the limiting cases I and II can be greater than those in the de Sitter inflation. For the nonplanar high-momentum modes, the amplitudes of the trispectra in both the limiting cases I and II can be at least comparable to those in the de Sitter inflation. Thus, combined with the direction dependence, the trispectra in all the limiting cases for the nonplanar high-momentum modes, as well as those for the planar modes, would be interesting to distinguish models.

\section{SUMMARY}

We have investigated the higher order spectra of a scalar field induced by the higher order time-derivative interactions, which becomes dominant above the energy scale $M$, in the universe with a primordial anisotropy. Along the lines of our recent works [10, 11], a change in the vacuum causes new features in the higher order spectra. We have evaluated the leading order contributions to the bispectra and trispectra.

The leading order contribution to the bispectra is given by the three-point interaction. The mixing of the negative frequency mode gives peaks of the bispectra in the limits of $k_{1} \rightarrow k_{2}+k_{3}$ and its permutation, where $k_{i}=\left|\mathbf{k}_{i}\right|$ are the magnitudes of the momentum vectors of a triangle. An imporant feature of the bispectra is that in the limit of $k_{3} \rightarrow k_{1}+k_{2}$ they are suppressed to that in the limit of $k_{1}=k_{2} \gg k_{3}$ by the ratio $\frac{k_{3}}{k}$, in fixing the total momentum $k=k_{1}+k_{2}+k_{3}$. For the nonplanar high-momentum modes, for various shapes of the triangle the ratio to the case of the de Sitter inflation is given by $\frac{M^{3}}{H_{0}^{3}} \epsilon_{*}^{4}$, which is larger than unity for $\frac{M}{H_{0}}>\epsilon_{*}^{-\frac{4}{3}}$, where $\epsilon_{*}$ is the adiabaticity parameter evaluated at the instance of the matching. However, this bound is not consistent with $\frac{M}{H_{0}}<\epsilon_{*}^{-1}$, which is obtained from the requirement that the time when the momentum of a mode becomes smaller than the energy scale of the derivative interactions becomes later than the time when the de Sitter mode function starts to become valid. For the planar modes, the bispectra are always larger than in the de Sitter inflation by a factor $\left(\frac{M}{H_{0}}\right)^{3}<\epsilon_{*}^{-3}$. In summary, for the nonplanar high-momentum modes the bispectra cannot be as large as that of de Sitter inflation. On the other hand, for the planar modes, they can be greater than those in the de Sitter inflation. The bispectra are direction dependent, and the dependence is significantly different in various limiting cases. Thus the bispectra for the planar modes would be particularly interesting to distinguish models.

We have also investigated the trispectra in the same spacetime background. The leading order contribution to the trispectra is obtained from two kinds of diagrams: the contact interaction and the scalar exchange interaction diagrams (see, e.g., [19] for the Feynman diagrams). We have first considered the nonplanar high-momentum modes. The trispectrum from the contact interaction is amplified in the limit of $k_{1} \rightarrow k_{2}+k_{3}+k_{4}$ (and in its permutation), compared to the case of the de Sitter inflation. The ratio of the trispectrum in the limit of $k_{3} \rightarrow k_{1}+k_{2}+k_{3}$ in the 
initially anisotropic universe to that in the isotropic one is given by $\frac{M^{5}}{H_{0}^{5}} \epsilon_{*}^{4}$, which is larger than unity for $\frac{M}{H_{0}}>\epsilon_{*}^{-\frac{4}{5}}$. This bound can be consistent with $\frac{M}{H_{0}}<\epsilon_{*}^{-1}$. Thus the trispectrum in this limit can be enhanced for smaller energy scales $M$ than in the case of the bispectrum. In the other important limits, its ratio becomes of order $\frac{M^{5}}{H_{0}^{5}} \epsilon_{*}^{7}$, which is larger than unity for $\frac{M}{H_{0}}>\epsilon_{*}^{-\frac{7}{5}}$. However, this bound cannot be consistent with $\frac{M}{H_{0}}<\epsilon_{*}^{-1}$. Thus it is hard to enhance these shapes of the trispectra. For the planar modes, the trispectra from the contact interactions are always larger than in the de Sitter inflation by a factor $\left(\frac{M}{H_{0}}\right)^{5}<\epsilon_{*}^{-5}$. In summary, for the nonplanar high-momentum modes, the trispectra can be as large as that of de Sitter inflation only for the limit of $k_{1} \rightarrow k_{2}+k_{3}+k_{4}$ (and also for its permutations). On the other hand, for the planar modes, these can be greater than those in the de Sitter inflation. The trispectra are direction dependent, and its dependence is significantly different in various limiting cases. Thus for the nonplanar high-momentum modes, the trispectra in this limiting case would be particularly interesting to distinguish models. Also, as for the bispectra, in all the limiting cases the planar modes would be important.

In the case of the trispectrum from the scalar exhange interactions, the amplitude in the the limit of $k_{1}+k_{2} \rightarrow$ $\left|\mathbf{k}_{1}+\mathbf{k}_{2}\right|$ and $k_{3}+k_{4} \rightarrow\left|\mathbf{k}_{1}+\mathbf{k}_{2}\right|$ (and also for $k_{1}+k_{4} \rightarrow\left|\mathbf{k}_{1}+\mathbf{k}_{4}\right|$ and $k_{2}+k_{3} \rightarrow\left|\mathbf{k}_{1}+\mathbf{k}_{4}\right|$ ) can be more important than that in the limit of $k_{1}=k_{2} \gg\left|\mathbf{k}_{1}+\mathbf{k}_{2}\right|$ and $k_{3}=k_{4} \gg\left|\mathbf{k}_{1}+\mathbf{k}_{2}\right|$, by the ratio $\frac{k}{k_{12}}$. This result is in contrast to the case of the bispectra and would give an unique signature from an initially anisotropic universe. For the nonplanar high-momentum modes, the ratio of the trispectra in the initially anisotropic universe to those in the case of the de Sitter inflation is given by $\frac{M^{6}}{H_{0}^{6}} \epsilon_{*}^{6}$, which can be more important for $\frac{M}{H_{0}}>\epsilon_{*}^{-1}$. This bound can be marginally consistent with $\frac{M}{H_{0}}<\epsilon_{*}^{-1}$. For the planar modes, the trispectra from the contact interactions are always larger than in the de Sitter inflation by a factor $\left(\frac{M}{H_{0}}\right)^{6}<\epsilon_{*}^{-6}$. In summary, for the nonplanar high-momentum modes the trispectra can be as large as that in de Sitter inflation in all the limiting cases. Also, for the planar modes, they can be greater than those in the de Sitter inflation. The trispectra are direction dependent, and its dependence is significantly different in various limiting cases. Thus for the nonplanar high-momentum modes, the trispectra in all the limiting cases would be particularly interesting to distinguish models. Also, as for the bispectra, in all the limiting cases the planar modes would be important.

Before closing this article, we would like to mention the directions of our future studies. One of the most important directions is to formulate the nonlinear cosmological perturbations theory in the anisotropic universe and evaluate the higher order spectra. Since in the anisotropic cosmological background, even at the level of the linear perturbations, there is a significant mixture of the different types of perturbations in terms of three-dimensional symmetry, the same effects may also become important during the isotropization, as well as in the change of the vacuum state. Another important direction is to cure the initial curvature singularity in other types of initially anisotropic spacetime which is singular, and to present a successful quantization scheme of the perturbations. They may be achieved by the matter fields, which could significantly backreact on the spacetime geometry and regularize the initial singularity.

\section{Acknowledgements}

We thank H-c. Kim for fruitful discussions. This work was supported by Yukawa fellowship and by Grant-in-Aid for Young Scientists (B) of JSPS Research, under Contract No. 24740162.

\section{Appendix A: Higher order spectra of a scalar field in the de Sitter universe}

In this appendix, as a reference, we discuss the higher spectra of a scalar field in the de Sitter universe. induced by the derivative interactions given in the main text (34).

\section{Bispectrum}

The bispectrum from the contact interaction in the de Sitter inflation is given by

$$
B\left(\mathbf{k}_{1}, \mathbf{k}_{2}, \mathbf{k}_{3}\right)=-\frac{3 H_{0}^{5}}{\left(k_{1} k_{2} k_{3}\right)\left(k_{1}+k_{2}+k_{3}\right)^{3}} .
$$




\section{Trispectrum}

a. Contribution from the contact interaction

The trispectrum from the contact interaction in the de Sitter inflation is given by

$$
T_{c}\left(\mathbf{k}_{1}, \mathbf{k}_{2}, \mathbf{k}_{3}, \mathbf{k}_{4}\right)=\frac{72 H_{0}^{8}}{\left(k_{1} k_{2} k_{3} k_{4}\right)\left(k_{1}+k_{2}+k_{3}+k_{4}\right)^{5}} .
$$

b. Contribution from the scalar exchange interaction diagram

The trispectrum from the scalar exchange interaction diagram in the de Sitter inflation is given by

$$
T_{s}\left(\mathbf{k}_{1}, \mathbf{k}_{2}, \mathbf{k}_{3}, \mathbf{k}_{4}\right)=\frac{9 H_{0}^{8}}{2\left(k_{1} k_{2} k_{3} k_{4}\right)}\left\{\frac{k_{12}}{\left(k_{1}+k_{2}+k_{12}\right)^{3}\left(k_{3}+k_{4}+k_{12}\right)^{3}}+\frac{k_{14}}{\left(k_{2}+k_{3}+k_{14}\right)^{3}\left(k_{1}+k_{4}+k_{14}\right)^{3}}\right\} .
$$

\section{References}

[1] E. Komatsu et al. [WMAP Collaboration], Astrophys. J. Suppl. 180, 330 (2009) arXiv:0803.0547 [astro-ph]].

[2] J. Dunkley et al. [WMAP Collaboration], Astrophys. J. Suppl. 180, 306 (2009) arXiv:0803.0586 [astro-ph]].

[3] C. J. Copi, D. Huterer, D. J. Schwarz and G. D. Starkman, Adv. Astron. 2010, 847541 (2010) arXiv:1004.5602 [astroph.CO]].

[4] A. de Oliveira-Costa, M. Tegmark, M. Zaldarriaga and A. Hamilton, Phys. Rev. D 69, 063516 (2004) arXiv:astro-ph/0307282

[5] C. Armendariz-Picon and L. Pekowsky, Phys. Rev. Lett. 102, 031301 (2009) arXiv:0807.2687 [astro-ph]]; T. S. Pereira and L. R. Abramo, Phys. Rev. D 80, 063525 (2009) arXiv:0907.2340 [astro-ph]];

[6] N. E. Groeneboom, L. Ackerman, I. K. Wehus and H. K. Eriksen, Astrophys. J. 722, 452 (2010) arXiv:0911.0150 [astroph.CO]]; A. Pontzen and H. V. Peiris, Phys. Rev. D 81, 103008 (2010) arXiv:1004.2706 [astro-ph.CO]].

[7] C. Pitrou, T. S. Pereira and J. P. Uzan, JCAP 0804, 004 (2008) arXiv:0801.3596 [astro-ph]];

[8] A. E. Gümrükçüoğlu, C. R. Contaldi and M. Peloso, JCAP 0711, 005 (2007) arXiv:0707.4179 [astro-ph]].

[9] A. E. Gümrükçüoğlu, L. Kofman and M. Peloso, Phys. Rev. D 78, 103525 (2008) arXiv:0807.1335 [astro-ph]].

[10] H. C. Kim and M. Minamitsuji, Phys. Rev. D 81, 083517 (2010) [Erratum-ibid. D 82, 109904 (2010)] arXiv:1002.1361 [gr-qc]].

[11] H. -C. Kim, M. Minamitsuji, JCAP 1103, 038 (2011). arXiv:1101.0329 [gr-qc]].

[12] A. Dey, S. Paban, arXiv:1106.5840 [hep-th]].

[13] R. M. Wald, Phys. Rev. D 28, 2118 (1983).

[14] X. Chen, Adv. Astron. 2010, 638979 (2010) arXiv:1002.1416 [astro-ph.CO]].

[15] K. Koyama, Class. Quant. Grav. 27, 124001 (2010). arXiv:1002.0600 [hep-th]].

[16] T. S. Bunch, P. C. W. Davies, Proc. Roy. Soc. Lond. A360, 117-134 (1978).

[17] R. Holman and A. J. Tolley, JCAP 0805, 001 (2008) arXiv:0710.1302 [hep-th]];

[18] S. Weinberg, Phys. Rev. D72, 043514 (2005). hep-th/0506236.

[19] X. Chen, B. Hu, M. -x. Huang, G. Shiu, Y. Wang, JCAP 0908, 008 (2009). arXiv:0905.3494 [astro-ph.CO]].

[20] P. Creminelli, JCAP 0310, 003 (2003). arXiv:astro-ph/0306122 [astro-ph]]. 